\title{
Strata of $k$-differentials
}

\author{
Matt Bainbridge, Dawei Chen, Quentin Gendron, Samuel Grushevsky \\ and Martin Möller
}

\begin{abstract}
A $k$-differential on a Riemann surface is a section of the $k$ th power of the canonical line bundle. Loci of $k$-differentials with prescribed number and multiplicities of zeros and poles form a natural stratification of the moduli space of $k$-differentials. In this paper, we give a complete description for the compactification of the strata of $k$-differentials in terms of pointed stable $k$-differentials, for all $k$. The upshot is a global $k$-residue condition that can also be reformulated in terms of admissible covers of stable curves. Moreover, we study properties of $k$-differentials regarding their deformations, residues, and flat geometric structure.
\end{abstract}

\section{Introduction}

Let $X$ be a smooth complex curve of genus $g$, and let $K_{X}$ be the canonical line bundle on $X$. For a positive integer $k$, a non-zero $k$-differential $\xi$ on $X$ is a non-trivial section of $K_{X}^{k}$; namely, $\xi$ can be written locally as $f(z)(d z)^{k}$, where $f(z)$ is a (possibly meromorphic) function of a local coordinate $z$, and the expression is invariant under change of coordinates. Abelian and quadratic differentials, corresponding to the cases $k=1$ and $k=2$, respectively, exhibit fascinating geometry that arises from associated flat structure and $\mathrm{SL}_{2}(\mathbb{R})$-action - we refer to the surveys [Zor06, Wri15, Che17] for these topics and recent advances.

Let $\mu=\left(m_{1}, \ldots, m_{n}\right)$ be a tuple of integers such that their sum is $k(2 g-2)$-we call $\mu$ a partition of $k(2 g-2)$ (allowing non-positive entries). If a $k$-differential $\xi$ has exactly $n$ zeros and poles of respective orders $m_{1}, \ldots, m_{n}$, we say that $\xi$ is of type $\mu$. Denote by $\Omega^{k} \mathcal{M}_{g}$ the $k$ th Hodge bundle over $\mathcal{M}_{g}$ whose fiber over a smooth genus $g$ curve $X$ is the space $H^{0}\left(X, K_{X}^{k}\right)$ of holomorphic $k$-differentials on $X$. The spaces of holomorphic $k$-differentials of all possible zero types form a stratification of $\Omega^{k} \mathcal{M}_{g}$ (and for the meromorphic case, one needs to twist by the polar part to define the $k$ th Hodge bundle; see Section 3.2 for more details). In this sense, denote by $\Omega^{k} \mathcal{M}_{g}(\mu)$ the stratum parameterizing $k$-differentials of type $\mu$. More precisely, $\Omega^{k} \mathcal{M}_{g}(\mu)$ parameterizes $\left(X, \xi, z_{1}, \ldots, z_{n}\right)$, where $X$ is a smooth genus $g$ curve, $z_{1}, \ldots, z_{n}$ are distinct marked points, and $\xi$ is a $k$-differential with zeros and poles at the $z_{i}$ such that $\operatorname{ord}_{z_{i}} \xi=m_{i}$. In this

Received 1 January 2018, accepted in final form 9 September 2018.

2010 Mathematics Subject Classification 30F30, 14H10 (primary), 32J05, 14H15 (secondary).

Keywords: $k$-differentials, strata, deformation, compactification, flat geometry.

This journal is (C) Foundation Compositio Mathematica 2019. This article is distributed with Open Access under the terms of the Creative Commons Attribution Non-Commercial License, which permits non-commercial reuse, distribution, and reproduction in any medium, provided that the original work is properly cited. For commercial re-use, please contact the Foundation Compositio Mathematica.

Research of the first author is supported in part by the Simons Foundation grant \#359821. Research of the second author is supported in part by the National Science Foundation under the CAREER grant DMS-13-50396 and a von Neumann Fellowship at the Institute for Advanced Study in Spring 2019. Research of the fourth author was supported in part by the National Science Foundation under the grant DMS-15-01265 and by a Simons Fellowship in Mathematics (Simons Foundation grant \#341858). 


\section{STRATA OF $k$-DIFFERENTIALS}

paper, we study the geometry of $\Omega^{k} \mathcal{M}_{g}(\mu)$ and, in particular, its compactification. Note that for $X$ smooth, $\xi$ determines its zeros and poles, but we mark these points as it will help us study degenerations of $\xi$.

There are similarities, but also fundamental differences, between the cases $k=1, k=2$, and $k \geqslant 3$. Since the $k$ th roots of unity are real only for $k \leqslant 2$, precisely in these cases the $\mathrm{SL}_{2}(\mathbb{R})$ action on the strata is well defined by varying the corresponding flat structure. Technically, however, the case $k=1$ is further different from $k \geqslant 2$. First, the dimension formula is different in the case of holomorphic abelian differentials compared to all the other cases (see Theorem 1.1 below). Moreover, the global residue condition, which is the upshot in the characterization of the strata compactification, differs significantly for $k=1$ and for any $k \geqslant 2$ (compare Definitions 1.2 and 1.4).

Recall that period coordinates provide local coordinates for the strata of abelian and quadratic differentials [HM79]. In particular, the existence of period coordinates implies the smoothness of the strata in these cases and gives the dimension of the strata. We will show that period coordinates can also be used for the strata of $k$-differentials for all $k$. Our first result is as follows.

THEOREM 1.1. Every connected component of the stratum $\Omega^{k} \mathcal{M}_{g}(\mu)$ is a smooth orbifold. If the component parameterizes $k$ th powers of holomorphic abelian differentials, it has dimension $2 g-1+n$. Otherwise, it has dimension $2 g-2+n$.

This theorem in particular implies that every connected component of $\Omega^{k} \mathcal{M}_{g}(\mu)$ is irreducible. For $k=1$, the strata of abelian differentials can have up to three connected components, caused by hyperelliptic and spin structures; see [KZ03]. We remark that for $k>1$, the strata of $k$ differentials can have more connected components due to another reason-for a common divisor $d$ of $m_{1}, \ldots, m_{n}$ and $k$, the locus of $d$ th powers of $(k / d)$-differentials of type $\mu / d$ provides connected components for $\Omega^{k} \mathcal{M}_{g}(\mu)$ (see Section 2 for more details).

For the question of compactifying $\Omega^{k} \mathcal{M}_{g}(\mu)$, we follow the same setup as in the case of $k=1$ treated in [BCGGM18]. First, consider the case when $\mu=\left(m_{1}, \ldots, m_{n}\right)$ is of holomorphic type, that is, when $m_{i} \geqslant 0$ for all $i$. Consider the pullback $\Omega^{k} \mathcal{M}_{g, n}$ of $\Omega^{k} \mathcal{M}_{g}$ from $\mathcal{M}_{g}$ to the moduli space $\mathcal{M}_{g, n}$ of $n$-pointed genus $g$ curves. Then $\Omega^{k} \mathcal{M}_{g, n}$ extends to a vector bundle $\Omega^{k} \overline{\mathcal{M}}_{g, n}$ over the Deligne-Mumford compactification $\overline{\mathcal{M}}_{g, n}$, which parameterizes pointed stable $k$-differentials. More precisely, let $f: \mathcal{C} \rightarrow \overline{\mathcal{M}}_{g, n}$ be the universal curve and $\omega_{f}$ the relative dualizing sheaf. Then we have $\Omega^{k} \overline{\mathcal{M}}_{g, n}=f_{*}\left(\omega_{f}^{k}\right)$, which is a direct generalization of the total space of the Hodge bundle in the case $k=1$. Taking the closure of the projectivized stratum $\mathbb{P} \Omega^{k} \mathcal{M}_{g}(\mu)$ in the projective bundle $\mathbb{P} \Omega^{k} \overline{\mathcal{M}}_{g, n}$, parameterizing $k$-differentials up to scaling by a non-zero complex number, we thus obtain a natural compactification $\mathbb{P} \Omega^{k} \overline{\mathcal{M}}_{g, n}^{\text {inc }}(\mu)$, called the incidence variety compactification of the projectivized stratum $\mathbb{P} \Omega^{k} \mathcal{M}_{g}(\mu)$. If $m_{i}<0$ for some $i$, we twist $\omega_{f}^{k}$ by the polar part of $\mu$ and then carry out the same construction (see Section 3 for more details).

As in the case $k=1$ treated in [BCGGM18], the idea of the incidence variety compactification is to record the limit location of zeros and poles on those components where the limit differential vanishes identically and to keep track of the relative scale of those components where the limit differential is non-zero.

Our characterization of the boundary points of $\mathbb{P} \Omega^{k} \overline{\mathcal{M}}_{g, n}^{\text {inc }}(\mu)$ is in terms of twisted $k$-differentials compatible with a full order (also called a level graph) on the set of irreducible components of a pointed stable curve. Simply speaking, a twisted $k$-differential $\eta=\left\{\eta_{v}\right\}$ of type $\mu$ on a nodal 


\section{BAINBRIDGE ET AL.}

curve $X$ is a collection of $k$-differentials on the irreducible components $X_{v}$ of $X$ satisfying the following conditions: (0) vanishing on the smooth locus of $X$ as prescribed by $\mu$, (1) matching zero and pole orders at each node, and (2) matching residues at poles of order $k$ (see Definition 3.2 for more details). This definition of twisted $k$-differentials is a direct generalization of the case $k=1$ in [BCGGM18].

To state the extra compatibility conditions that characterize twisted $k$-differentials that lie in the boundary of $\mathbb{P} \Omega^{k} \overline{\mathcal{M}}_{g, n}^{\text {inc }}(\mu)$, we first recall how this characterization works for abelian differentials, that is, for the case $k=1$.

Let $\Gamma$ be the dual graph of a nodal curve $X$. A full order on the irreducible components of $X$ is a relation $\succcurlyeq$ on the set $V$ of vertices of $\Gamma$ that is reflexive, transitive, and such that for any two vertices $v_{1}$ and $v_{2}$, at least one of the statements $v_{1} \succcurlyeq v_{2}$ and $v_{2} \succcurlyeq v_{1}$ holds. Remark that any map $\ell: V \rightarrow \mathbb{R}$ assigning real numbers to vertices of $\Gamma$ defines a full order on $\Gamma$ by setting $v_{1} \succcurlyeq v_{2}$ if and only if $\ell\left(v_{1}\right) \geqslant \ell\left(v_{2}\right)$. We call a dual graph $\Gamma$ equipped with a full order on its vertices a level graph and denote it by $\bar{\Gamma}$. An edge is called horizontal if it joins two vertices at the same level and called vertical otherwise. If $e$ is an edge between $X_{v_{1}} \succ X_{v_{2}}$, then the corresponding points that are glued to form the node $e$ are denoted by $q_{e}^{+}$on $X_{v_{1}}$ and $q_{e}^{-}$on $X_{v_{2}}$. Finally, we denote by $X_{>L}$ the subcurve of $X$ consisting of all irreducible components $X_{v}$ such that $\ell(v)>L$. See Section 3.3 for more details of these notions. We now recall the definition of twisted abelian differentials $(k=1)$ compatible with a level graph.

Definition 1.2 ([BCGGM18]). Let $\left(X, z_{1}, \ldots, z_{n}\right)$ be a stable $n$-pointed curve with dual graph $\Gamma$, and let $\bar{\Gamma}$ be a full order on $\Gamma$. A twisted abelian differential $\eta$ of type $\mu$ on $X$ is called compatible with $\bar{\Gamma}$ if it satisfies the following conditions:

(3) (Partial order) If a node identifies $q_{1} \in X_{v_{1}}$ with $q_{2} \in X_{v_{2}}$, then $v_{1} \succcurlyeq v_{2}$ if and only if $\operatorname{ord}_{q_{1}} \eta_{v_{1}} \geqslant-1$. Moreover, $v_{1} \asymp v_{2}$ if and only if $\operatorname{ord}_{q_{1}} \eta_{v_{1}}=-1$.

(4-ab) (Global residue condition for abelian differentials) For every level $L$ and every connected component $Y$ of $X_{>L}$ that does not contain a marked point with a prescribed pole (that is, there is no $z_{i} \in Y$ with $\left.m_{i}<0\right)$, the following condition holds. Let $\left\{q_{1}, \ldots, q_{b}\right\}$ denote the set of all nodes where $Y$ intersects $X_{=L}$. Then

$$
\sum_{j=1}^{b} \operatorname{Res}_{q_{j}^{-}} \eta_{v^{-}\left(q_{j}\right)}=0,
$$

where we recall that $q_{j}^{-} \in X_{=L}$ and $v^{-}\left(q_{j}\right)$ is a vertex of $\bar{\Gamma}_{=L}$.

The main result of [BCGGM18] characterizes the boundary of the incidence variety compactification of strata of abelian differentials in terms of twisted abelian differentials compatible with level graphs.

For general $k$, the first idea is to lift a twisted $k$-differential via a certain covering construction and reduce the problem to the case of abelian differentials on the covering curve. While this is easy to state, the resulting condition $(\widehat{4})$ (see Definition 1.3) formulated this way depends on the choice of the cover and is thus less direct to check in an explicit example. We thus proceed to give an alternative characterization of the closure via a condition (4) (see Definition 1.4) imposed directly on the twisted $k$-differential, without passing to a cover. The resulting statement is more elaborate but is verifiable directly on the curve without investigating the set of all suitable covers.

For the approach via the covering construction, we proceed as follows. For a $k$-differential $\xi$ on a smooth connected curve $X$, there exists a canonical cover $\pi: \widehat{X} \rightarrow X$, cyclic of degree $k$, 


\section{StRATA OF $k$-DIFFERENTIALS}

such that $\pi^{*} \xi=\omega^{k}$ for an abelian differential $\omega$ on $\widehat{X}$ and such that $\tau^{*} \omega=\zeta \omega$, where $\tau$ is a deck transformation of order $k$ and $\zeta$ is a primitive $k$ th root of unity. If $X$ is nodal and $\eta$ is a twisted $k$-differential on $X$, then one can first carry out the canonical covering construction over each irreducible component of $X$ and then glue them to form an admissible cover $\pi: \widehat{X} \rightarrow X$ of a nodal curve (in the sense of admissible covers; see [HM98, Section 3.G]). If there exists a twisted abelian differential $\widehat{\omega}$ on $\widehat{X}$ such that $\pi^{*} \eta=\widehat{\omega}^{k}$ and $\tau^{*} \widehat{\omega}=\zeta \widehat{\omega}$, we say that $(\pi: \widehat{X} \rightarrow X, \widehat{\omega})$ is a normalized cover. See Sections 2.1 and 4 for more details on canonical covers of smooth curves and normalized covers of nodal curves, respectively.

Given an admissible cover $\pi: \widehat{X} \rightarrow X$ with dual graphs $\widehat{\Gamma}$ and $\Gamma$, a full order $\bar{\Gamma}$ can be lifted via $\pi$ to define a full order $\widehat{\bar{\Gamma}}$, which we call the lifted level graph. Now we can formulate the conditions needed to describe the closure of the stratum of $k$-differentials.

Definition 1.3. Let $\left(X, z_{1}, \ldots, z_{n}\right) \in \overline{\mathcal{M}}_{g, n}$ be a pointed stable curve, and let $\bar{\Gamma}$ be a level graph on $X$. A twisted $k$-differential $\eta$ of type $\mu$ on $X$ is called compatible with $\bar{\Gamma}$ if it satisfies the following conditions:

(3) (Partial order) If a node of $X$ identifies $q_{1} \in X_{v_{1}}$ with $q_{2} \in X_{v_{2}}$, then $v_{1} \succcurlyeq v_{2}$ if and only if $\operatorname{ord}_{q_{1}} \eta_{v_{1}} \geqslant-k$. Moreover, $v_{1} \asymp v_{2}$ if and only if $\operatorname{ord}_{q_{1}} \eta_{v_{1}}=-k$.

(4) ( $\widehat{G}$ lobal residue condition) There exists a normalized cover $(\widehat{X}, \widehat{\omega})$ of $(X, \eta)$ such that the twisted abelian differential $\widehat{\omega}$ satisfies the global residue condition (4-ab) in Definition 1.2 with respect to the lifted level graph $\widehat{\bar{\Gamma}}$.

The above definition of compatibility is in terms of the existence of a normalized cover satisfying certain conditions. By a graph-theoretic argument studying the combinatorics of all possible normalized covers, we will show that it is equivalent to a condition phrased purely in terms of the twisted $k$-differential $\eta$.

In order to state this alternative condition of compatibility, we need the notion of the $k$-residue $\operatorname{Res}_{z}^{k} \xi$ for a $k$-differential $\xi$ with a pole at $z$ of order an integer multiple of $k$. This $k$-residue is defined to be the $k$ th power of the coefficient of the degree $(-1)$ term of a meromorphic abelian differential $\omega$ at $z$ such that locally $\xi=\omega^{k}$ (see Proposition 3.1). Note that unless the order of the pole of $\xi$ at $z$ is equal to $k$, the $k$-residue does not have to be equal to the coefficient of $z^{-k}(d z)^{k}$ in the Laurent expansion of $\xi$ at $q$. Since such a meromorphic abelian differential is only defined up to multiplication by a $k$ th root of unity, there will be some symmetrization taking place, and hence we introduce the following symmetric polynomial $P_{n, k}$ in $n$ variables:

$$
P_{n, k}\left(R_{1}, \ldots, R_{n}\right):=\prod_{\left\{\left(r_{1}, \ldots, r_{n}\right) \mid r_{i}^{k}=R_{i}\right\}} \sum_{i=1}^{n} r_{i},
$$

where the product is taken over all $n$-tuples of complex numbers $\left\{r_{1}, \ldots, r_{n}\right\}$ such that $r_{i}^{k}=R_{i}$ for all $i$. As $P_{n, k}$ is symmetric with respect to the $k$ th roots of $R_{i}$, it is indeed a polynomial in $R_{i}$.

We are now ready to state the global $k$-residue condition. In order to understand it better, we first point out that the global residue condition (4-ab) imposed by the preimage of a connected component $Y$ of $X_{>L}$ at level $L$ is automatically satisfied if the normalized cover above $Y$ has fewer than $k$ components (see Proposition 5.1). This can happen in two cases. The first case is when the restriction of $\eta$ to any irreducible component $X_{v}$ of $Y$ is not the $k$ th power of an abelian differential, which leads to condition ii) below. The other case is when the restriction of $\eta$ on every irreducible component of $Y$ is the $k$ th power of an abelian differential but it is possible to 


\section{BAINBRIDGE ET AL.}

make some "criss-cross" when identifying the nodes of the cover, which leads to conditions iii) and iv) below (see Example 4.3 for an illustration of "criss-cross").

Definition 1.4. Let $\left(X, z_{1}, \ldots, z_{n}\right) \in \overline{\mathcal{M}}_{g, n}$ be a pointed stable curve, $\bar{\Gamma}$ a level graph on $X$, and $\eta$ a twisted $k$-differential of type $\mu$ on $X$ satisfying condition (3) of Definition 1.3. We then define the following condition:

(4) (Global k-residue condition) For every level $L$ and every connected component $Y$ of $\Gamma_{>L}$, one of the following cases holds:

i) The component $Y$ contains a marked pole.

ii) The component $Y$ contains a vertex $v$ such that $\eta_{v}$ is not a $k$ th power of a (possibly meromorphic) abelian differential.

iii) (Horizontal criss-cross in $Y$ ) For every vertex $v$ of $Y$, the $k$-differential $\eta_{v}$ is the $k$ th power of an abelian differential $\omega_{v}$. Moreover, for every choice of a collection of $k$ th roots of unity $\left\{\zeta_{v}: v \in Y\right\}$, there exists a horizontal edge $e$ in $Y$ where the differentials $\left\{\zeta_{v} \omega_{v}\right\}_{v \in Y}$ do not satisfy the matching residue condition.

iv) (Vertical criss-cross in $Y$ ) For every vertex $v$ of $Y$, the $k$-differential $\eta_{v}$ is the $k$ th power of an abelian differential $\omega_{v}$. Moreover, there exist a level $K>L$ and a collection of $k$ th roots of unity $\left\{\zeta_{e}: e \in E\right\}$ indexed by the set $E$ of non-horizontal edges $e$ of $Y$ whose lower end lies in $Y_{=K}$, such that the following two conditions hold. First, there exists a directed simple loop $\gamma$ in the dual graph of $Y_{\geqslant K}$ such that

$$
\prod_{e \in \gamma \cap E} \zeta_{e}^{ \pm 1} \neq 1
$$

where the sign of the exponent is \pm 1 according to whether $\gamma$ passes through $e$ in upward or downward direction. Second, for every connected component $T$ of $Y_{>K}$, the equation

$$
\sum_{e \in E_{T}} \zeta_{e} \operatorname{Res}_{q_{e}^{-}} \omega_{v^{-}(e)}=0
$$

holds, where $E_{T}$ is the subset of edges in $E$ such that their top vertices lie in $T$.

v) ( $Y$ imposes a residue condition) The $k$-residues at the edges $e_{1}, \ldots, e_{N}$ joining $Y$ to $\Gamma_{=L}$ satisfy the equation

$$
P_{N, k}\left(\operatorname{Res}_{q_{e_{1}}^{-}}^{k} \eta_{v^{-}\left(e_{1}\right)}, \ldots, \operatorname{Res}_{q_{e_{N}}^{-}}^{k} \eta_{v^{-}\left(e_{N}\right)}\right)=0,
$$

where $P_{N, k}$ is defined by $(1.1)$.

This seemingly complicated condition (4) is imposed directly on $(X, \eta)$, unlike the condition $(\widehat{4})$ that is in terms of the existence of a suitable normalized cover. We point out that one needs to check conditions iv) or v) only if conditions i), ii), and iii) do not hold, in which case the zero and pole orders of $\eta$ at the nodes of $Y$ are all divisible by $k$, and thus the notion of $k$-residue and consequently the residue condition are not trivial.

We will prove in Section 5 that for a twisted $k$-differential satisfying conditions (0)-(3), conditions $(\widehat{4})$ and (4) are equivalent, in other words, that a twisted $k$-differential is compatible with $\bar{\Gamma}$ if and only if it satisfies conditions (0)-(3) and (4).

Finally, we can state our main theorem that describes the incidence variety compactification of the strata of $k$-differentials. 


\section{StRATA OF $k$-DIFFERENTIALS}

TheOREM 1.5. A pointed stable $k$-differential $\left(X, \xi, z_{1}, \ldots, z_{n}\right)$ is contained in the incidence variety compactification $\mathbb{P} \Omega^{k} \overline{\mathcal{M}}_{g, n}^{\text {inc }}(\mu)$ of a stratum $\mathbb{P} \Omega^{k} \mathcal{M}_{g}(\mu)$ if and only if the following conditions hold:

(i) There exists a full order $\bar{\Gamma}$ on $X$ such that its maxima are the irreducible components $X_{v}$ of $X$ on which $\xi$ is not identically zero.

(ii) There exists a twisted $k$-differential $\eta$ of type $\mu$ on $X$, compatible with $\bar{\Gamma}$.

(iii) On every irreducible component $X_{v}$ where $\xi$ is not identically zero, $\eta_{v}=\left.\xi\right|_{X_{v}}$.

The space of twisted differentials by itself will play a central role in upcoming work by the authors to construct a smooth compactification of strata of abelian differentials and $k$-differentials. The following dimension statement is the basic reason that spaces of twisted differentials (or rather finite covers of them) can be used as orbifold charts for such a smooth compactification.

Let $\bar{\Gamma}$ be a level graph, and let $h$ be the number of horizontal edges of $\bar{\Gamma}$. Let $\Omega^{k, \text { ab }} \mathcal{M}_{g}(\mu)$ be the union of the components of $\Omega^{k} \mathcal{M}_{g}(\mu)$ consisting of $k$ th powers of holomorphic abelian differentials and $\Omega^{k, \text { non-ab }} \mathcal{M}_{g}(\mu)$ the union of the other components.

THEOREM 1.6. The space of twisted abelian differentials of type $\mu$ compatible with $\bar{\Gamma}$ either is empty or has pure dimension equal to $\operatorname{dim} \Omega \mathcal{M}_{g}(\mu)-h$.

For any $k \geqslant 2$, the space of twisted $k$-differentials of type $\mu$ compatible with $\bar{\Gamma}$ that are smoothable into $\Omega^{k, \text { non-ab }} \mathcal{M}_{g}(\mu)$ either is empty or has pure dimension equal to $\operatorname{dim} \Omega^{k, \text { non-ab }} \mathcal{M}_{g}(\mu)-h$.

The same result holds when we replace "non-ab" with "ab" in the above theorem; its proof reduces to the abelian case. See Section 6 for more detailed definitions and statements.

This paper is organized as follows. In Section 2, we study tangent spaces of the strata of $k$-differentials and prove Theorem 1.1. We also establish period coordinates in general and describe $k$-differentials from the viewpoint of flat geometry. In Section 3, we study the notion of $k$-residues and introduce in detail the ambient space of pointed stable $k$-differentials that we use for the incidence variety compactification. In Sections 4, we prove Theorem 1.5 in the case when condition $(\widehat{4})$ of Definition 1.3 is satisfied. In Section 5, we show the equivalence of condition $(\widehat{4})$ and condition (4) of Definition 1.4 by a graph-theoretic argument. In Section 6 , we prove Theorem 1.6 by a homological argument. Finally, in Section 7, we provide some examples that are consequences of the incidence variety compactification, and we also outline a flat geometric approach for proving our main result.

\section{Deformations of $k$-differentials}

The goal of this section is to study the local structure of the strata of $k$-differentials. We identify the tangent spaces of the strata with a hypercohomology group and compute its dimension in Theorem 2.1. As in the well-studied cases of abelian and quadratic differentials (that is, $k=1$ and $k=2$ ), in Theorem 2.2 we exhibit period coordinates for the strata of $k$-differentials. In the course of the proof, we introduce the key notion of canonical covers, which will be used throughout the paper. We also explain the flat geometric meaning of $k$-differentials in terms of a $(1 / k)$-translation structure, which we will use to present examples.

Let us first introduce the major results of this section. Denote by $\operatorname{div}(\xi)=\sum_{i=1}^{n} m_{i} z_{i}$ the underlying divisor of a $k$-differential $\xi$ on a smooth curve $X$. If $E=\sum_{j} m_{j} p_{j}$ is any divisor on $X$ with non-zero integer coefficients $m_{j}$, we let $E_{\text {red }}=\sum_{j} p_{j}$ be the corresponding reduced divisor, 


\section{BAINBRIDGE ET AL.}

and we decompose it as $E_{\text {red }}=Z-P$ with

$$
Z=\sum_{m_{j}>-k} p_{j} \text { and } P=\sum_{m_{j} \leqslant-k} p_{j},
$$

into its "zero" and "pole" divisor. We stress that the poles of order $m_{i}>-k$ of a $k$-differential $\xi$ contribute to the zero part of $\operatorname{div}(\xi)_{\text {red }}$ (in the sense of passing to the canonical $k$-cover). In the result below, we show that the tangent space of the stratum at $\xi$ can be identified with the hypercohomology of the following two-term complex of sheaves in degrees zero and one:

$$
\mathcal{C}^{\bullet}\left(\mathcal{L}_{\xi}\right)=\mathcal{L}_{\xi}: T_{X}\left(-\operatorname{div}(\xi)_{\text {red }}\right) \rightarrow K_{X}^{k}(-\operatorname{div}(\xi)),
$$

where $\mathcal{L}_{\xi}$ is the $k$-Lie derivative associated with $\xi$, whose definition we will recall in Section 2.2.

ThEOREM 2.1. Let $\left(X, \xi, z_{1}, \ldots, z_{n}\right) \in \Omega^{k} \mathcal{M}_{g}\left(m_{1}, \ldots, m_{n}\right)$ be a (possibly meromorphic) $k$-differential on a smooth curve $X$. Then the tangent space of the stratum at $\xi$ can be identified as

$$
T_{\left(X, \xi, z_{1}, \ldots, z_{n}\right)} \Omega^{k} \mathcal{M}_{g}\left(m_{1}, \ldots, m_{n}\right)=H^{1}\left(X, \mathcal{C}^{\bullet}\left(\mathcal{L}_{\xi}\right)\right) .
$$

Moreover, the connected component of $\Omega^{k} \mathcal{M}_{g}\left(m_{1}, \ldots, m_{n}\right)$ containing $\left(X, \xi, z_{1}, \ldots, z_{n}\right)$ is a smooth orbifold of dimension $2 g-1+n$ if $\xi$ is the $k$ th power of a holomorphic abelian differential and of dimension $2 g-2+n$ otherwise.

As mentioned in the introduction, for a divisor $d$ of $k$, the loci of $d$ th powers of $(k / d)$ differentials can provide connected components for the strata of $k$-differentials. A $k$-differential is called primitive if it is not the $d$ th power of a $(k / d)$-differential for any divisor $d>1$ of $k$. For local computations, it suffices to focus on the case of primitive $k$-differentials, because otherwise we may pass to the case of $(k / d)$-differentials instead.

Given a $k$-differential $\xi$ on $X$, there exists a canonical cover $\pi: \widehat{X} \rightarrow X$, which is cyclic of degree $k$, such that the pullback of $\xi$ is the $k$ th power of an abelian differential $\omega$ on $\widehat{X}$. Moreover, there exists a deck transformation $\tau$ of $\pi$ of order $k$ such that $\tau^{*} \omega=\zeta \omega$, where $\zeta$ is a chosen primitive $k$ th root of unity (fixed from now on for the rest of the paper). A canonical cover together with such a deck transformation is called normalized and denoted by the pair $(\pi, \tau)$.

We remark that $\widehat{X}$ is connected if and only if $\xi$ is primitive. In that case, the deck transformation group of $\pi$ is cyclic of order $k$, generated by $\tau$. If $\xi$ is not primitive, then $\widehat{X}$ is disconnected, and $\tau$ only generates a cyclic subgroup of order $k$ in the deck transformation group. The construction of a canonical cover and its properties will be explained in Section 2.1.

Let $\widehat{E}$ be the $\pi$-pullback of $\operatorname{div}(\xi)$; we denote the $\pi$-preimage of the poles $P$ and zeros $Z$ by $\widehat{P}$ and $\widehat{Z}$, respectively. Let $\widehat{\imath}: \widehat{X} \backslash \widehat{P} \rightarrow \widehat{X}$ and $\widehat{\jmath}: \widehat{X} \backslash \widehat{E}_{\text {red }} \rightarrow \widehat{X} \backslash \widehat{P}$ be the inclusion maps. We can describe period coordinates for $\Omega^{k} \mathcal{M}_{g}\left(m_{1}, \ldots, m_{n}\right)$ using the cohomology on $\widehat{X}$ as follows.

TheOREM 2.2. Let $\xi$ be a primitive $k$-differential in $\Omega^{k} \mathcal{M}_{g}\left(m_{1}, \ldots, m_{n}\right)$. The tangent space $T_{\left(X, \xi, z_{1}, \ldots, z_{n}\right)} \Omega^{k} \mathcal{M}_{g}\left(m_{1}, \ldots, m_{n}\right)$ is isomorphic to the $\tau$-eigenspace of $H^{1}\left(\widehat{X}, \widehat{\imath}_{*}\left(\widehat{\jmath}_{!} \mathbb{C}\right)\right)$ for the eigenvalue $\zeta$.

As a consequence of the above theorem, we exhibit period coordinates geometrically for the strata.

Corollary 2.3. Let $V_{\zeta}$ be the eigenspace of $H_{1}(\widehat{X} \backslash \widehat{P}, \widehat{Z} ; \mathbb{C})$ associated with the eigenvalue $\zeta$. Locally at the primitive $k$-differential $\xi$, the stratum $\Omega^{k} \mathcal{M}_{g}\left(m_{1}, \ldots, m_{n}\right)$ has coordinates given by the periods $\int_{\gamma_{i}} \omega$, where $\left\{\gamma_{i}\right\}$ is a basis of $V_{\zeta}$ and $\pi^{*} \xi=\omega^{k}$ for an abelian differential $\omega$ on $\widehat{X}$. 


\section{StRATA OF $k$-DIFFERENTIALS}

We remark that Theorem 2.1 is a generalization of [Möl08, Theorem 2.3] and [Mon17, Proposition 3.1] from the case of abelian differentials to $k$-differentials. Recently, Mondello (personal communication) and Schmitt [Sch18] have also independently obtained this result. Theorem 2.2 generalizes the known case of quadratic differentials, originally studied by Hubbard-Masur [HM79] and Veech [Vee86].

\subsection{The canonical cover}

We begin by constructing a cyclic cover of $X$ such that the pullback of $\xi$ is the $k$ th power of an abelian differential. A reference for this material is [EV92, Section 3]. We remark that here a cover means a possibly ramified and disconnected cover.

Recall that $\zeta$ is a fixed primitive $k$ th root of unity. We group the zeros and poles of $\xi$ according to their remainders $\bmod k$, so that

$$
\operatorname{div}(\xi)=\sum_{j=0}^{k-1} \sum_{i \in I_{j}} m_{i, j} z_{i, j}, \quad \text { where } I_{j}=\left\{i \in\{1, \ldots, n\}: m_{i} \equiv j \bmod k\right\},
$$

and we write the coefficients as $m_{i, j}=k \ell_{i, j}+j$. Next, we define a line bundle $\mathcal{L}$ and the remainder $D$ of $\operatorname{div}(\xi) \bmod k$ :

$$
\mathcal{L}=K_{X}\left(-\sum_{j} \sum_{i \in I_{j}} l_{i, j} z_{i, j}\right) \quad \text { and } \quad D=\sum_{j=0}^{k-1} \sum_{i \in I_{j}} j z_{i, j} .
$$

Then $\xi$ can be considered as a section $s$ of the line bundle $\mathcal{L}^{k}$, with zero divisor $D$. The triple $(\mathcal{L}, s, D)$ is the defining datum of a degree $k$ cyclic cover $\pi: \widehat{X} \rightarrow X$, given by providing the $\mathcal{O}_{X}$-module $\oplus_{i=0}^{k-1} \mathcal{L}^{-i}$ with an algebra structure by mapping a section $\alpha$ of $\mathcal{L}^{-k}$ to $s \alpha \in \mathcal{O}_{X}$ and normalizing the resulting cover, as explained, for example, in [EV92, Section 3.5]. We say that this cover is the canonical cover of $X$ induced by $\xi$. This algebraic viewpoint makes it obvious that the construction of the canonical cover works for a family of smooth curves $f: \mathcal{X} \rightarrow B$ over some base $B$, provided with a family of $k$-differentials, that is, a section of $f_{*}\left(\omega_{f}^{k}\right)$, where $\omega_{f}$ is the relative dualizing sheaf of the family.

There is an alternative geometric viewpoint of this construction (see, for example, [Lan04] for the case $k=2$ ). One first constructs an unramified cover $\widehat{X}^{0}$ of $X^{0}=X \backslash \operatorname{div}(\xi)_{\text {red }}$ as follows. Cover $X^{0}$ by open sets $U_{\alpha}$ and take $k$ patches $U_{\alpha, i} \cong U_{\alpha}$ for $i=0, \ldots, k-1$. We provide $U_{\alpha_{i}}$ with a flat structure given by the abelian differential $\omega_{\alpha, i}=\left.\zeta^{i} \xi\right|_{U_{\alpha}} ^{1 / k}$, for some choice of a $k$ th root of $\xi$ over $U_{\alpha}$. Note that different choices simply permute the labeling of the $U_{\alpha, i}$, so that the construction in the end will not depend on these choices. Whenever $U_{\alpha}$ and $U_{\beta}$ intersect in $X^{0}$, we glue the patches $U_{\alpha, i}$ and $U_{\beta, j}$ for the indices $i$ and $j$ such that $\varphi_{\alpha \beta}^{*} \omega_{\alpha, i}=\omega_{\beta, j}$ to form $\widehat{X}^{0}$, where $\varphi_{\alpha \beta}$ is the transition function from $U_{\beta}$ to $U_{\alpha}$. Finally, the unramified cover $\widehat{X}^{0}$ extends uniquely to a (possibly ramified) cover $\pi: \widehat{X} \rightarrow X$, and the local differentials $\omega_{\alpha, i}$ glue to a global differential $\omega$ on $\widehat{X}$ such that $\omega^{k}=\pi^{*} \xi$.

For both constructions, it is clear that $\omega$ is an eigenform of a deck transformation $\tau$ such that $\tau^{*} \omega=\zeta \omega$, and hence $(\pi, \tau)$ is a normalized cover. Moreover, if $\widehat{X}$ has $r$ connected components, then the restrictions of $\omega$ to two connected components differ by a non-trivial power of $\zeta$.

To compute the divisor of $\omega$, we let $r_{i, j}=\operatorname{gcd}\left(m_{i, j}, k\right)=\operatorname{gcd}(j, k)$ and $d_{i, j}=k / r_{i, j}$. Then in the geometric construction, the fiber of $\pi$ over $z_{i, j}$ consists of $r_{i, j}$ distinct points $z_{i, j}^{(1)}, \ldots, z_{i, j}^{\left(r_{i, j}\right)}$, 


\section{BAinbridge ET AL.}

each with multiplicity $d_{i, j}$; that is, the ramification divisor of $\pi$ is

$$
R=\sum_{i, j, \ell}\left(d_{i, j}-1\right) z_{i, j}^{(\ell)}
$$

Now, we can summarize the properties of a canonical cover.

Proposition 2.4. The algebraic and geometric constructions of a canonical cover give isomorphic degree $k$ cyclic covers $\pi: \widehat{X} \rightarrow X$. Moreover, there exists an abelian differential $\omega$ on $\widehat{X}$ such that $\omega^{k}=\pi^{*} \xi$, where such an $\omega$ is unique up to multiplication by a $k$ th root of unity on each connected component of $\widehat{X}$.

The canonical cover is connected if and only if $\xi$ is a primitive $k$-differential. In this case, the genus $\widehat{g}$ of $\widehat{X}$ is

$$
\widehat{g}=1+k(g-1)+\frac{1}{2}\left(k n-\sum_{i, j} r_{i, j}\right),
$$

and the underlying divisor of $\omega$ is

$$
\operatorname{div}(\omega)=\sum_{i, j, \ell}\left(d_{i, j}-1+\frac{d_{i, j} m_{i, j}}{k}\right) z_{i, j}^{(\ell)}=\sum_{i, j, \ell}\left(\frac{k-r_{i, j}+m_{i, j}}{r_{i, j}}\right) z_{i, j}^{(\ell)} .
$$

Proof. The algebraic construction gives a section $\widehat{s}$ of $\pi^{*} \mathcal{L}$ such that $\widehat{s}^{k}=\pi^{*} s$, and the associated divisor of $\widehat{s}$ is $\sum_{i, j, \ell}\left(j / r_{i, j}\right) z_{i, j}^{(\ell)}$, as one can see from the following interpretation (cf. [EV92, Remark 3.14b)]). If $\mathbb{V}\left(\mathcal{L}^{-k}\right)$ is the geometric line bundle associated with $\mathcal{L}^{k}$, then global sections of $\mathcal{L}^{k}$ are geometric sections of $\mathbb{V}\left(\mathcal{L}^{-k}\right)$. There is a natural symmetric power map $\rho: \mathbb{V}\left(\mathcal{L}^{-1}\right) \rightarrow$ $\mathbb{V}\left(\mathcal{L}^{-k}\right)$ of geometric bundles over $X$, and $\widehat{X}=\rho^{-1}(s(X))$ by construction. Consequently, $\widehat{X}$ comes with a natural map to $\mathbb{V}\left(\mathcal{L}^{-1}\right)$ and hence also with a map to its pullback to $\widehat{X}$. Since $K_{\widehat{X}}=\pi^{*} K_{X} \otimes \mathcal{O}_{\widehat{X}}(R)$, we can interpret $\widehat{s}$ as a section $\omega$ of $K_{\widehat{X}}$ with $\omega^{k}=\pi^{*} \xi$.

Taking the $\rho$-preimage of a section of $\mathbb{V}\left(\mathcal{L}^{-k}\right)$ is equivalent to locally gluing $k$ th roots of the section in the unique consistent way, as in the geometric construction. This implies that the two constructions agree away from the branch points and hence also agree across the branch points, as guaranteed by the uniqueness of a smooth extension.

The connectedness claim is shown in [EV92, Lemma 3.15]. The expressions of the genus and the divisor of $\pi^{*} \xi$ follow from the Riemann-Hurwitz formula, since we have already determined the branching orders of $\pi$. In fact, from the geometric construction of the canonical cover, it is obvious that $\operatorname{div}(\omega)=(1 / k) \operatorname{div}\left(\pi^{*} \xi\right)$, and from the algebraic construction, $\operatorname{div}(\omega)$ is equivalently given by $R+\sum_{i, j, \ell}\left(d_{i, j} \ell_{i, j}+\left(j / r_{i, j}\right)\right) z_{i, j}^{(\ell)}$.

Finally, note that if $\widetilde{\omega}$ is another abelian differential on $\widehat{X}$ with $\widetilde{\omega}^{k}=\pi^{*} \xi$, then $\widetilde{\omega} / \omega$ is a function on $\widehat{X}$ whose $k$ th power is 1 . Hence, this function is equal to a $k$ th root of unity on every connected component of $\widehat{X}$.

\subsection{The Lie derivative}

The Lie derivative $\mathrm{L}_{v}$ associated with a vector field $v$ applied to a symmetric tensor $T$ is defined as $\mathrm{L}_{v}=\left.\frac{\partial}{\partial t} \varphi_{t}^{*} T\right|_{t=0}$, where $\varphi_{t}$ is the one-parameter group of diffeomorphisms generated by $v$. Axiomatically, one can describe the collection of maps $\mathrm{L}_{v}: K_{X}^{k} \rightarrow K_{X}^{k}$ for all $k \in \mathbb{Z}$ by requiring that

(i) $\mathrm{L}_{v}$ is the directional derivative for $k=0$; 


\section{StRATA OF $k$-DIFFERENTIALS}

(ii) it satisfies the Leibniz rule

$$
\mathrm{L}_{v}(S \otimes T)=\mathrm{L}_{v}(S) \otimes T+S \otimes \mathrm{L}_{v}(T)
$$

for tensor products and the analogous Leibniz rule for contractions;

(iii) $L_{v}$ commutes with exterior derivative on functions.

We need the Lie derivative with $v$ as argument instead, that is, we define

$$
\mathcal{L}_{\xi}: T_{X}\left(-\operatorname{div}(\xi)_{\text {red }}\right) \rightarrow K_{X}^{k}(-\operatorname{div}(\xi)), \quad \mathcal{L}_{\xi}(v):=\mathrm{L}_{v}(\xi)
$$

to be the $k$-Lie derivative associated with $\xi \in H^{0}\left(X, K_{X}^{k}\right)$. Using the axioms given above, we find by induction on $k$ that in the local coordinate $z$ where $\xi=a(z)(d z)^{k}$, the map $\mathcal{L}_{\xi}$ is given by

$$
\mathcal{L}_{\xi}\left(b(z) \frac{\partial}{\partial z}\right)=\left(a^{\prime} b+k a b^{\prime}\right)(d z)^{k},
$$

which is the main property we need further on.

Proof of Theorem 2.1. We abbreviate $E=\operatorname{div}(\xi)$. The first-order deformations of a smooth pointed curve $\left(X, z_{1}, \ldots, z_{n}\right)$ are given by one-cocycles $\left\{D_{\alpha \beta}\right\}$ with values in $T_{X}\left(-E_{\text {red }}\right)$ for a cover of $X$ by open sets $\left\{U_{\alpha}\right\}$. Let $\varphi_{\alpha \beta}$ denote the transition function on the intersection $U_{\alpha} \cap U_{\beta}$. We determine over which of these deformations we can extend the $k$-differential $\xi$. A deformation of $\xi$ is locally of the form

$$
\widetilde{\xi}_{\alpha}=\xi_{\alpha}+\epsilon \xi_{\alpha}^{\prime}=f_{\alpha}\left(u_{\alpha}\right)\left(d u_{\alpha}\right)^{k}+\epsilon g_{\alpha}\left(u_{\alpha}\right)\left(d u_{\alpha}\right)^{k},
$$

where $\epsilon^{2}=0$, and we require that $\operatorname{ord}_{p}\left(\xi_{\alpha}^{\prime}\right) \geqslant \operatorname{ord}_{p}\left(\xi_{\alpha}\right)$ at every point $p \in X_{\alpha}$ for deformations that preserve the type $\left(m_{1}, \ldots, m_{n}\right)$ of the $k$-differential. Moreover, we have

$$
\begin{aligned}
\varphi_{\alpha \beta}^{*}\left(\widetilde{\xi}_{\alpha}\right) & =\left[f_{\alpha}\left(u_{\alpha}+\epsilon D_{\alpha \beta}\left(u_{\alpha}\right)\right)+\epsilon g_{\alpha}\left(u_{\alpha}\right)\right]\left(d\left(u_{\alpha}+\epsilon D_{\alpha \beta}\left(u_{\alpha}\right)\right)\right)^{k} \\
& =\xi_{\alpha}+\epsilon\left[\left(f_{\alpha}^{\prime} D_{\alpha \beta}+k f_{\alpha} D_{\alpha \beta}^{\prime}\right)\left(d u_{\alpha}\right)^{k}+\xi_{\alpha}^{\prime}\right] \\
& =\xi_{\alpha}+\epsilon\left[\mathcal{L}_{\xi_{\alpha}}\left(D_{\alpha \beta}\right)+\xi_{\alpha}^{\prime}\right] .
\end{aligned}
$$

Hence, the pairs $\left\{\left(D_{\alpha \beta}, \xi_{\alpha}^{\prime}\right)\right\} \in C^{1}\left(\left\{U_{\alpha}\right\}, T_{X}\left(-E_{\text {red }}\right)\right) \oplus C^{0}\left(\left\{U_{\alpha}\right\}, K_{X}^{k}(-E)\right)$ glue to a first-order deformation of $\left(X, \xi, z_{1}, \ldots, z_{n}\right)$ if and only if $\varphi_{\alpha \beta}^{*}\left(\widetilde{\xi}_{\alpha}\right)-\widetilde{\xi}_{\beta}=0$, that is, $\mathcal{L}_{\xi_{\alpha}}\left(D_{\alpha \beta}\right)+\xi_{\alpha}^{\prime}-$ $\xi_{\beta}^{\prime}=0$ for all $\alpha, \beta$. Moreover, the trivial deformations are given by the trivial deformations of the curve and the deformations such that $\mathcal{L}_{\xi_{\alpha}}\left(D_{\alpha \beta}\right)+\xi_{\alpha}^{\prime}=0$ at the level of the differential. Hence, the deformations of $(X, \xi)$ are parameterized by the first hypercohomology group of the complex $\mathcal{C}^{\bullet}\left(\mathcal{L}_{\xi}\right)$.

The filtration of the complex in the middle of the diagram

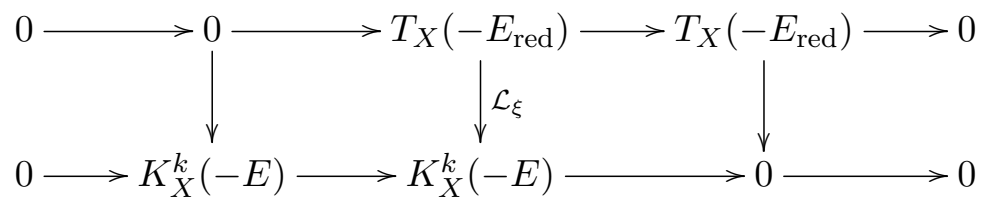

gives a short exact sequence of two-term complexes. The associated long exact sequence of cohomology is

$$
\begin{aligned}
0 & \longrightarrow H^{0}\left(X, T_{X}\left(-E_{\text {red }}\right)\right) \stackrel{\beta_{0}}{\longrightarrow} H^{0}\left(X, K_{X}^{k}(-E)\right) \stackrel{\gamma_{0}}{\longrightarrow} H^{1}\left(X, \mathcal{C}^{\bullet}\left(\mathcal{L}_{\xi}\right)\right) \\
& \stackrel{\alpha_{1}}{\longrightarrow} H^{1}\left(X, T_{X}\left(-E_{\text {red }}\right)\right) \stackrel{\beta_{1}}{\longrightarrow} H^{1}\left(X, K_{X}^{k}(-E)\right) \stackrel{\gamma_{1}}{\longrightarrow} H^{2}\left(X, \mathcal{C}^{\bullet}\left(\mathcal{L}_{\xi}\right)\right) \longrightarrow 0 .
\end{aligned}
$$




\section{BAINBRIDGE ET AL.}

The Riemann-Roch formula implies that $h^{1}\left(X, \mathcal{C}^{\bullet}\left(\mathcal{L}_{\xi}\right)\right)=2 g-2+n+h^{2}\left(X, \mathcal{C}^{\bullet}\left(\mathcal{L}_{\xi}\right)\right)$. We want to compute $H^{2}\left(X, \mathcal{C}^{\bullet}\left(\mathcal{L}_{\xi}\right)\right)$, which coincides with the cokernel of the map

$$
\beta_{1}: H^{1}\left(X, T_{X}\left(-E_{\text {red }}\right)\right) \rightarrow H^{1}\left(X, K_{X}^{k}(-E)\right) .
$$

By Serre duality, $H^{2}\left(X, \mathcal{C} \bullet\left(\mathcal{L}_{\xi}\right)\right)$ is dual to the kernel of the map

$$
\beta_{1}^{\vee}=H^{0}\left(\mathcal{L}_{\xi}^{\vee}\right): H^{0}\left(X, K_{X}^{1-k}(E)\right) \rightarrow H^{0}\left(X, K_{X}^{2}\left(E_{\text {red }}\right)\right),
$$

where $\mathcal{L}_{\xi}^{\vee}: K_{X}^{1-k}(E) \rightarrow K_{X}^{2}\left(E_{\text {red }}\right)$ is the dual map of $\mathcal{L}_{\xi}$. We compute this dual map $\mathcal{L}_{\xi}^{\vee}$ in local coordinates. Let $\tau$ and $v$ be local sections of $K_{X}^{1-k}(E)$ and $T_{X}\left(-E_{\text {red }}\right)$, respectively. We fix a local coordinate $z$ on an open subset $U$ such that

$$
\xi=a(z)(d z)^{k}, \quad \tau=c(z)\left(\partial_{z}\right)^{k-1}, \quad v=b(z) \partial_{z} .
$$

By equation (2.5), we have

$$
\left\langle c\left(\partial_{z}\right)^{k-1}, \mathcal{L}_{\xi}(v)\right\rangle=\int_{U} c\left(a^{\prime} b+k a b^{\prime}\right) d z=-\int_{U} b\left((k-1) a^{\prime} c+k a c^{\prime}\right) d z
$$

since $d(k a b c)=k\left(a^{\prime} b c+a b^{\prime} c+a b c^{\prime}\right)$. The dual of the $k$-Lie derivative is thus locally given by $\mathcal{L}_{\xi}^{\vee}(\tau)=-\left((k-1) a^{\prime} c+k a c^{\prime}\right)(d z)^{2}$. Denote by $\Lambda$ the kernel of the map $\mathcal{L}_{\xi}^{\vee}$, which is given by those $\tau \in H^{0}\left(X, K_{X}^{1-k}(E)\right)$ that satisfy the differential equation $(k-1) a^{\prime} c+k a c^{\prime}=0$ locally everywhere. The solutions to this equation are simply given by $c=r a^{(1-k) / k}$, where $r$ is a constant in $\mathbb{C}$. Therefore, the kernel $\Lambda$ is at most one-dimensional. If $a^{1 / k}$ is not globally defined, that is, if $\xi$ is not the $k$ th power of an abelian differential, then $\Lambda$ is clearly zero. If $\xi=\omega^{k}$ for an abelian differential $\omega$ on $X$, then $E=k \operatorname{div}(\omega)$ and $\operatorname{div}\left(a^{(1-k) / k}\right)=(1-k) \operatorname{div}(\omega)$. If $\omega$ is strictly meromorphic, then $r$ has to be zero, for otherwise $\operatorname{div}(c)+E=\operatorname{div}(\omega)$ is not effective, contradicting that $c$ is a section of $\mathcal{O}_{X}(E)$; hence, in this case, $\Lambda$ is also zero. If $\omega$ is holomorphic, the same argument implies that $r$ can be any constant; hence, $\Lambda$ is one-dimensional. Summarizing the above discussion, we have shown that $h^{2}\left(X, \mathcal{C}^{\bullet}\left(\mathcal{L}_{\xi}\right)\right)$ is 1 if $\xi$ is the $k$ th power of a holomorphic abelian differential and is zero otherwise. This implies that the dimension of the tangent space of $\Omega^{k} \mathcal{M}_{g}\left(m_{1}, \ldots, m_{n}\right)$ at $(X, \xi)$ is $2 g-1+n$ if $\xi$ is the $k$ th power of a holomorphic abelian differential and is $2 g-2+n$ otherwise.

The smoothness of the strata of abelian differentials is known; hence, we assume that $\xi$ is not the $k$ th power of an abelian differential. We want that the deformation induced by the cocycle $\left\{\left(D_{\alpha \beta}, \xi_{\alpha}^{\prime}\right)\right\}$ of $\mathcal{C}^{\bullet}\left(\mathcal{L}_{\xi}\right)$ extends to a higher-order deformation. It is a classical fact that the deformation of the complex structure induced by $\left\{D_{\alpha \beta}\right\}$ extends to higher order if and only if its coboundary in $H^{2}\left(X, T_{X}\right)$ vanishes (see, for example, [Kod05]). Similarly, we obtain that the deformation given by the cocycle $\left\{\left(D_{\alpha \beta}, \xi_{\alpha}^{\prime}\right)\right\}$ extends if and only if its coboundary in $H^{2}\left(X, \mathcal{C} \bullet\left(\mathcal{L}_{\xi}\right)\right)$ vanishes. We have shown that the second hypercohomology group of $\mathcal{C}^{\bullet}\left(\mathcal{L}_{\xi}\right)$ is trivial when $\xi$ is not the $k$ th power of an abelian differential. This implies that the deformations of $(X, \xi)$ are unobstructed, and consequently the stratum is smooth at $(X, \xi)$.

Proof of Theorem 2.2 and Corollary 2.3. We start with the case when there are no poles of order $m_{i} \leqslant-k$, that is, $P=\emptyset$. For a singularity $p$ with $\operatorname{ord}_{p} \xi=m>-k$, one can choose a suitable local coordinate $z$ such that $\xi=a(z)(d z)^{k}=z^{m}(d z)^{k}$ locally at $p$, which corresponds to $z=0$ (see Proposition 3.1). By the definition of $E_{\text {red }}$, a local section $b(z) \frac{\partial}{\partial z}$ of $T_{X}\left(-E_{\text {red }}\right)$ at $p$ has the form $b(z)=\sum_{i=1}^{\infty} b_{i} z^{i}$. Then the Lie derivative $\mathcal{C}^{\bullet}\left(\mathcal{L}_{\xi}\right)$ maps $b$ to $a^{\prime} b+k a b^{\prime}=z^{m} \sum_{i=1}^{\infty}(m+k i) b_{i} z^{i-1}$, which can be any element in $z^{m} \mathbb{C}[[z]]$. It follows that $\mathcal{C}^{\bullet}\left(\mathcal{L}_{\xi}\right)$ is surjective in this case, and so the first hypercohomology of $\mathcal{L}_{\xi}$ is isomorphic to the first cohomology of $\Lambda_{k}=\operatorname{Ker}\left(\mathcal{L}_{\xi}\right)$. 


\section{StRATA OF $k$-DIFFERENTIALS}

This kernel is a $\mathbb{C}$-local system of rank one, generated outside of the zeros of $\xi$ by $a^{-1 / k}$ in the local coordinates used for (2.5). Since these $a^{-1 / k}$ correspond to the local patches used for the geometric construction of the canonical cover, it follows that $\Lambda_{k}$ is trivialized on $\widehat{X}$. More precisely, $\pi^{*} \Lambda_{k}=\widehat{\jmath} ! \mathbb{C}$, where $\widehat{\jmath}: \widehat{X} \backslash \widehat{E}_{\text {red }} \rightarrow \widehat{X}$ is the inclusion defined above. On the other hand, $H^{1}(\widehat{X}, \widehat{\jmath} ! \mathbb{C})=H^{1}\left(X, \pi_{*} \widehat{\jmath} ! \mathbb{C}\right)$ by the degeneration of the Leray spectral sequence, on which $\tau$ acts equivariantly. Moreover, $\pi_{*} \widehat{\jmath} ! \mathbb{C}$ splits as a direct sum of its $k$ eigenspaces for the action of $\tau$, each of which is a local system of rank one. Since there is a canonical injective map $\Lambda_{k} \rightarrow \pi_{*} \pi^{*} \Lambda_{k}=\pi_{*} \widehat{\jmath} ! \mathbb{C}$ and since $\Lambda_{k}$ is locally generated by the element $a^{-1 / k}$ in the eigenspace where $\tau$ acts by the $k$ th root of unity $\zeta$, we obtain that $H^{1}(\widehat{X}, \widehat{\jmath} ! \mathbb{C})^{\tau=\zeta}=H^{1}\left(X, \Lambda_{k}\right)$, as claimed.

In the general case when there are poles of orders $m_{i} \leqslant-k$, that is, $P \neq \emptyset$, recall that we defined the inclusions $\imath: X \backslash P \rightarrow X$ and $\jmath: X \backslash E_{\text {red }} \rightarrow X \backslash P$. The comparison between singular and sheaf cohomology and the trivial case of the Leray spectral sequence for maps of relative dimension zero gives the isomorphisms between the eigenspaces

$$
H_{1}(\widehat{X} \backslash \widehat{P}, \widehat{Z} ; \mathbb{C})_{\tau=\zeta}^{\vee}=H^{1}(\widehat{X} \backslash \widehat{P}, \widehat{\jmath} ! \mathbb{C})_{\tau=\zeta}=H^{1}\left(\widehat{X}, \widehat{\imath}_{*}(\widehat{\jmath} ! \mathbb{C})\right)_{\tau=\zeta}
$$

and the equivalence of the theorem and the corollary. Since the complex

$$
0 \rightarrow \Lambda_{k} \rightarrow \imath^{*}\left(T_{X}\left(-E_{\text {red }}\right)\right) \rightarrow \imath^{*}\left(K_{X}^{k}(-E)\right) \rightarrow 0
$$

on $X \backslash P$ is still exact, we deduce that

$$
\left.H^{1}(\widehat{X} \backslash \widehat{P}, \widehat{\jmath} ! \mathbb{C})_{\tau=\zeta}=H^{1}\left(X \backslash P, \Lambda_{k}\right)=H^{1}\left(X, \imath_{*} \Lambda_{k}\right)=H^{1}\left(X, \widetilde{\mathcal{C} \bullet\left(\mathcal{L}_{\xi}\right.}\right)\right),
$$

where $\widetilde{\mathcal{C}^{\bullet}\left(\mathcal{L}_{\xi}\right)}$ is the complex

$$
\left(\imath_{*} \imath^{*}\left(T_{X}\left(-E_{\mathrm{red}}\right)\right) \rightarrow \imath_{*} \imath^{*}\left(K_{X}^{k}(-E)\right)\right) \cong \imath_{*}\left(\mathcal{O}_{X \backslash P}\right) \otimes\left(T_{X}\left(-E_{\mathrm{red}}\right) \rightarrow K_{X}^{k}(-E)\right) .
$$

Since $\imath_{*}\left(\mathcal{O}_{X \backslash P}\right)$ is the sheaf of functions with arbitrary poles at points in $P$, there is an obvious inclusion of complexes $\mathcal{C}^{\bullet}\left(\mathcal{L}_{\xi}\right) \rightarrow \widetilde{\mathcal{C} \bullet\left(\mathcal{L}_{\xi}\right)}$. To conclude, we have to show that this inclusion induces an isomorphism on the first cohomology groups. The cokernel complex consists of the sum over all points $z_{j}$ with $m_{j} \leqslant-k$ of the Lie derivative maps on polar parts

$$
\mathbb{C}((z)) / z \mathbb{C}[z] \rightarrow \mathbb{C}((z)) / z^{m_{j}} \mathbb{C}[z]
$$

We claim that this map is surjective. In fact, for a singularity $p$ with $\operatorname{ord}_{p} \xi=m \leqslant-k$, one can choose a suitable local coordinate $z$ such that $a(z)=z^{m}$ if $k \nmid m$, such that $a(z)=r / z^{k}$ if $m=-k$, and such that $a(z)=\left(z^{m / k}+s / z\right)^{k}$ if $m<-k$ and $k \mid m$ (see Proposition 3.1). We give the details in the last case, the first two being easier. Write $m=\ell k$ with $\ell \leqslant-2$. For any $j \leqslant 0$ and $b=z^{j}$, we compute

$$
\begin{aligned}
a^{\prime} b+k a b^{\prime} & =\left(z^{\ell}+\frac{s}{z}\right)^{k-1} z^{j-1} k\left((\ell+j) z^{\ell}+(j-1) \frac{s}{z}\right) \\
& =k(\ell+j) z^{m+j-1}+\text { higher-order terms }
\end{aligned}
$$

which implies the surjectivity of (2.7). Consequently, the first (and of course also the second) cohomology of (2.7) vanishes, and the long exact sequence of hypercohomology groups implies the claim.

\section{$2.3(1 / k)$-translation structure}

Recall that abelian and quadratic differentials correspond to translation and half-translation surfaces; hence, they can be realized as plane polygons with certain edge identifications under 


\section{BAINBRIDGE ET AL.}

translation and rotation by $\pi$, respectively. The edges of the polygons as complex vectors correspond to period coordinates; hence, in these cases, it is geometrically clear that they control deformations of differentials in the respective strata.

In Corollary 2.3, we have established period coordinates for the strata of $k$-differentials for all $k$. We want to describe $k$-differentials by a certain generalized translation structure, so that period coordinates can be visible under the corresponding flat geometric presentation.

First, we define a building block that will help us describe the neighborhood of a singularity (under the induced flat metric) of a $k$-differential. Take $(1 / k)$ th of a disk in $\mathbb{C}$ under the standard Euclidean metric, that is, a sector bounded by an arc of angle $2 \pi / k$. We say that it is a $(1 / k)$ disk. Take $n$ copies of such $(1 / k)$-disks, each with two boundary rays $A_{i}$ and $B_{i}$, such that $B_{i}$ is identified with $A_{i+1}$ by translation for $i=1, \ldots, n-1$. The total angle at the center $p$ of the disks is $2 \pi n / k$. Hence, for identifying $B_{n}$ with $A_{1}$, we need a rotation by $2 \pi r / k$, where $r$ is the remainder of $n \bmod k$. In other words, the last identification is induced by $z \mapsto \zeta^{r} z+c$, where $\zeta=e^{2 \pi i / k}$ and $c$ is a constant - in particular, it is a translation if and only if $k \mid n$. After gluing these disks as above, we say that $p$ is a cone point of angle $2 \pi n / k$.

Now, we define a $(1 / k)$-translation surface as a closed topological surface $X$ with a finite set of points $E$ satisfying the following conditions:

- There is an atlas of charts $X \backslash E \rightarrow \mathbb{C}$ whose transition functions are of type $z \mapsto \zeta^{r} z+c$, where $r \in \mathbb{Z}$ and $c$ is a constant.

- For each $p \in E$, there is a homeomorphism of a neighborhood of $p$ to a neighborhood of a cone point of angle $2 \pi n / k$, which is an isometry away from $p$.

Proposition 2.5. There is a one-to-one correspondence between $(1 / k)$-translation surfaces and $k$-differentials whose pole orders are at most $k-1$. In particular, a singularity of a $k$-differential with order $m$ corresponds to a cone point of angle $2 \pi(k+m) / k$.

Proof. At a singularity $p$ of a $k$-differential $\xi$, we choose a suitable local coordinate $z$ such that $\xi=z^{m}(d z)^{k}$ (see Proposition 3.1 for the general statement and proof, noting that here we are dealing with poles of order at most $k-1)$. Rewrite it formally as $\left(z^{m / k} d z\right)^{k}$. Then a flat geometric neighborhood of $p$ can be identified with a gluing of $(k+m)$ copies of a $(1 / k)$-disk as above; hence, the claim follows.

As in the cases of abelian and quadratic differentials, one can equivalently describe $k$-differentials in terms of cutting and pasting polygons (see [Wri15, Definitions 1.1, 1.5, and 1.7]). In this sense, a $(1 / k)$-translation surface is a collection of polygons in $\mathbb{C}$ with edge identifications as follows:

- All the edges are grouped in pairs such that the edges in the same pair are identified by translation and rotation by $2 \pi n / k$ for some $n \in \mathbb{Z}$.

- For the edges in the same pair, the polygon interior nearby one edge does not map to the polygon interior nearby the other edge.

Two such collections of polygons define the same $(1 / k)$-translation surface if and only if one can be refined and re-glued to form the other by translations and rotations by $2 \pi n / k$ for some $n \in \mathbb{Z}$.

For meromorphic abelian differentials, the flat geometric neighborhood of a pole can be described by half-infinite cylinders or by gluing (broken) half-planes along their boundary via translation, as introduced by Boissy [Boi15]. Such a construction can similarly be carried out for 


\section{StRATA OF $k$-DIFFERENTIALS}

$k$-differentials with arbitrary pole orders. The building blocks for a pole of order at least $k$ are either half-infinite cylinders or (broken) $(1 / 2 k)$-planes, where the edge identification is given by translation and rotation by $2 \pi n / k$ for $n \in \mathbb{Z}$.

Finally, we provide an example to illustrate the flat geometric meaning of period coordinates in Corollary 2.3.

EXAMPLE 2.6. Take a primitive $\xi \in \Omega^{k} \mathcal{M}_{g}((2 g-2) k)$. Let $z$ be the unique zero of $\xi$. Since $k \mid(2 g-2) k$, the canonical cover $\pi: \widehat{X} \rightarrow X$ is unramified. Let $\tau$ be the deck transformation of $\widehat{X}$ corresponding to $\zeta$, and let $\widehat{z}_{1}, \ldots, \widehat{z}_{k}$ be the preimages of $z$ such that $\tau\left(\widehat{z}_{i}\right)=\widehat{z}_{i+1}$. By the Riemann-Hurwitz formula,

$$
2 \widehat{g}-2=k(2 g-2)
$$

hence, we have

$$
\operatorname{dim} H_{1}(\widehat{X}, \mathbb{C}) / \pi^{*} H_{1}(X, \mathbb{C})=2 \widehat{g}-2 g=(k-1)(2 g-2) .
$$

There is a basis $\widehat{\gamma}_{1}, \ldots, \widehat{\gamma}_{2 g-2}$ of absolute homology classes that belong to $V_{\zeta}$. For the relative part, take a path $\alpha_{i}$ that joins $\widehat{z}_{i}$ to $\widehat{z}_{i+1}$, for $i=1, \ldots, k$, such that $\tau\left(\alpha_{i}\right)=\alpha_{i+1}$ in $H_{1}\left(\widehat{X}, \widehat{z}_{1}, \ldots, \widehat{z}_{k} ; \mathbb{C}\right)$. Let

$$
\gamma_{2 g-1}=\sum_{i=1}^{k} \zeta^{-i} \alpha_{i}
$$

Then

$$
\tau^{*} \gamma_{2 g-1}=\sum_{i=1}^{k} \zeta^{-i} \alpha_{i-1}=\zeta \gamma_{2 g-1}
$$

hence, $\gamma_{2 g-1}$ is in $V_{\zeta}$ and lies in the relative part. Therefore, $\widehat{\gamma}_{1}, \ldots, \widehat{\gamma}_{2 g-2}, \gamma_{2 g-1}$ together form a basis of $V_{\zeta}$.

The case of $g=2$ and $k=3$ is pictured in Figure 1. The bottom surface represents a primitive cubic differential $\xi$ in $\Omega^{3} \mathcal{M}_{2}(6)$, where the edges with the same labels are identified by translation (for $a$ and $b$ ) and rotation by $2 \pi / 3$ (for $c$ and $d$ ). In particular, the three vertices of the inner triangle are identified as the same point which forms the unique zero of $\xi$. In the top three surfaces, edges with the same labels are identified by translation; hence, after gluing, we obtain an abelian differential $\omega$, and the resulting surface admits a canonical triple cover of the bottom which maps $a_{0}, a_{1}, a_{2}$ to $a$, and so on. Note that in this case, $\omega$ lies in the stratum $\Omega \mathcal{M}_{4}(2,2,2)$. The absolute part of the equivariant homology consists of weighted sums of the preimages of $a$ and of $b$ with weight pattern as in equation (2.8). The relative part is given by equation (2.8), where the $\alpha_{i}$ are the preimages of $c$.

In Section 7, we will use the (1/k)-translation surface viewpoint to present a number of examples highlighting various aspects of the incidence variety compactification that will be discussed soon.

\section{Stable $k$-differentials and $k$-residues}

In this section, we define in detail the ambient space that we will use for the incidence variety compactification. For this purpose, we need a local standard form for $k$-differentials and the notion of $k$-residues. We will also recall the definition of level graphs and twisted differentials as introduced in [BCGGM18]. 


\section{BAINBRidge ET AL.}

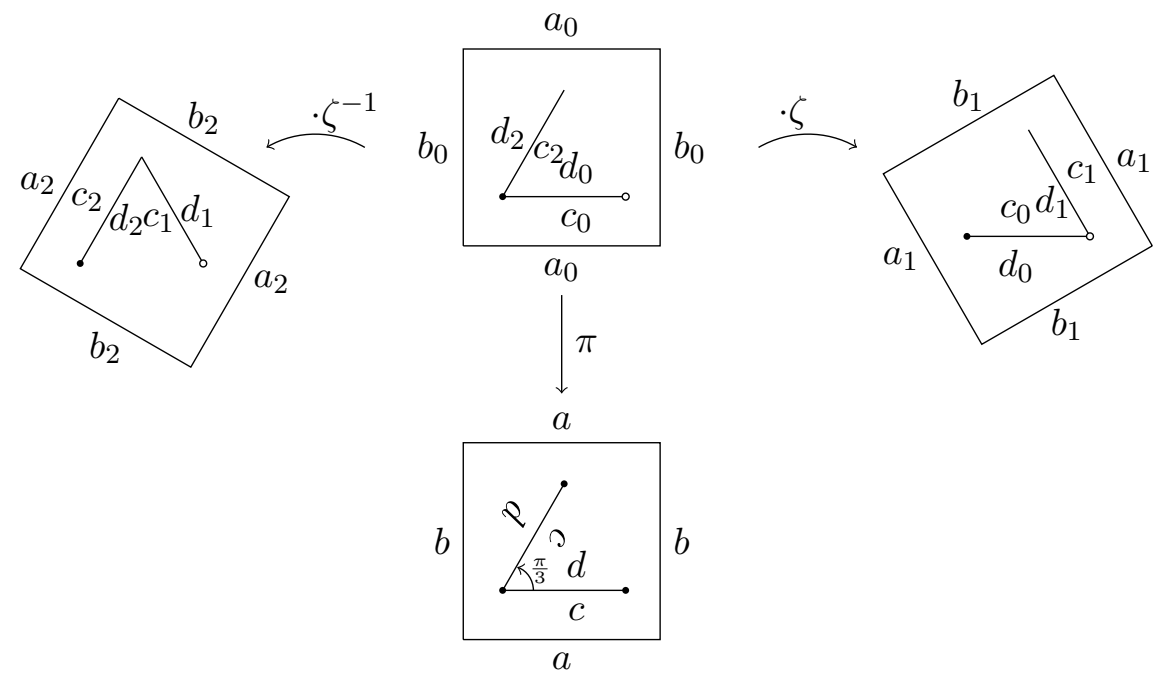

Figure 1. Canonical cover of a primitive cubic differential in $\Omega^{3} \mathcal{M}_{2}(6)$

\subsection{Standard coordinates for $\boldsymbol{k}$-differentials, and $\boldsymbol{k}$-residues}

Contrary to the case of abelian differentials, not every pole of a $k$-differential $\xi$ has a local invariant like the residue, independent of the choice of a local coordinate, but only poles of order divisible by $k$ have such an invariant. We make this precise in the following proposition.

Proposition 3.1. Given a $k$-differential $\xi$ on a sufficiently small disk $V$ around 0 , denote by $m$ the order $\operatorname{ord}_{0} \xi$. Then there exist a conformal map $\phi:\left(\Delta_{R}, 0\right) \rightarrow(V, 0)$ defined on a disk of sufficiently small radius $R$ and a number $r \in \mathbb{C}$ such that

$$
\phi^{*}(\xi)= \begin{cases}z^{m}(d z)^{k} & \text { if } m>-k \text { or } k \nmid m, \\ \frac{r}{z^{k}}(d z)^{k} & \text { if } m=-k, \\ \left(z^{m / k}+\frac{s}{z}\right)^{k}(d z)^{k} & \text { if } m<-k \text { and } k \mid m,\end{cases}
$$

where in the last case $s \in \mathbb{C}$ and $s^{k}=r$. The germ of $\phi$ is unique up to multiplication by an $(m+k)$ th root of unity when $m>-k$ or $k \nmid m$, and up to multiplication by a non-zero constant if $m=-k$.

We call the coordinate $z$ provided in the proposition the standard coordinate for $\xi$ and call the number $r$ the $k$-residue of $\xi$ and denote it by $r=\operatorname{Res}_{0}^{k} \xi$. For convenience, we also set $\operatorname{Res}_{0}^{k} \xi=0$ if $m>-k$ or $k \nmid m$. We remark that while for $m=-k$, the residue $\operatorname{Res}_{0}^{k} \xi$ is simply the order $-k$ term of the Laurent series of $\xi$ at 0 , for $m \neq-k$, the $k$-residue does not admit such an easy description.

For quadratic differentials (that is, $k=2$ ), this proposition was stated and proved in [Str84, Section 6]. The same argument works for general $k$ as well. For the reader's convenience, we include a proof.

Proof. Suppose that in a local coordinate $w$ around 0 , the $k$-differential $\xi$ is given by

$$
\xi=w^{m}\left(a_{0}+a_{1} w+\cdots\right)(d w)^{k},
$$




\section{StRATA OF $k$-DIFFERENTIALS}

with $a_{0} \neq 0$. Setting $w=\varphi(u)=u^{k}$, we obtain that

$$
\varphi^{*}(\xi)=u^{m k}\left(a_{0}+a_{1} u^{k}+\cdots\right)\left(k u^{k-1} d u\right)^{k} .
$$

Note that the $k$ th root of the power series $a_{0}+a_{1} u^{k}+\cdots$ is again a power series $b_{0}+b_{1} u^{k}+\cdots$ in $u^{k}$ with the property that

$$
\varphi^{*}(\xi)=\left(u^{m+k-1}\left(b_{0}+b_{1} u^{k}+\cdots\right) k d u\right)^{k} .
$$

Let $\psi$ be a coordinate change that puts the abelian differential inside the parenthesis on the right-hand side into the standard form. In the first case $m>-k$ or $m+k-1 \not \equiv-1(\bmod k)$, this abelian differential has no residue, and hence

$$
\psi^{*} \varphi^{*}(\xi)=\left(v^{m+k-1} d v\right)^{k}=\frac{1}{k^{k}} v^{m k}\left(d\left(v^{k}\right)\right)^{k} .
$$

Since this differential depends on $v^{k}$ only, setting $z=v^{k}$ provides the desired expression of the standard coordinate. Moreover, for $v$ to satisfy that $u^{m+k-1}\left(b_{0}+b_{1} u^{k}+\cdots\right) k d u=v^{m+k-1} d v$, it suffices to have $u^{m+k}\left(c_{0}+c_{1} u^{k}+\cdots\right)=v^{m+k}$ by integrating both sides, where $c_{0} \neq 0$. If we write $v=u y$, the condition reduces to $c_{0}+c_{1} u^{k}+\cdots=y^{m+k}$; hence, $y$ can be expressed in terms of $u^{k}$, which implies that $z=v^{k}=u^{k} y^{k}$ is a well-defined coordinate change from the original coordinate $w=u^{k}$.

If $m=-k$, the existence of standard coordinates for abelian differentials implies the existence of a $\psi$ such that

$$
\psi^{*} \varphi^{*}(\xi)=k^{k}\left(s v^{-1} d v\right)^{k}=r\left(v^{-k} d\left(v^{k}\right)\right)^{k} .
$$

Again, this is the pullback under $v \mapsto z=v^{k}$ of a $k$-differential which is of the form $\left(r / z^{k}\right)(d z)^{k}$. The last case of $m<-k$ and $k \mid m$ is similar, except for the more delicate point that now the abelian differential written in standard coordinate may have a residue.

From the flat geometric viewpoint of Section 2.3, the $k$-residue corresponds to a half-infinite cylinder for a pole of order $-k$ and to a half-infinite slit for poles of higher order (see the right of Figure 4). We stress that the width of the slit or of the cylinder represents some $k$ th root of the $k$-residue.

\subsection{Stable $k$-differentials}

Let $f: \mathcal{C} \rightarrow \overline{\mathcal{M}}_{g, n}$ be the universal curve over the Deligne-Mumford moduli space of $n$-pointed stable genus $g$ curves, and let $\omega_{f}$ be the relative dualizing sheaf. Consider the space $\Omega^{k} \overline{\mathcal{M}}_{g, n}:=$ $f_{*}\left(\omega_{f}^{k}\right)$ as a vector bundle over $\overline{\mathcal{M}}_{g, n}$. This is to say, its fibers are spaces $H^{0}\left(X, \omega_{X}^{k}\right)$ parameterizing stable $k$-differentials $\xi$ over pointed stable curves $\left(X, z_{1}, \ldots, z_{n}\right)$, where $\omega_{X}$ is the dualizing sheaf of $X$. We call points $\left(X, \xi, z_{1}, \ldots, z_{n}\right) \in \Omega^{k} \overline{\mathcal{M}}_{g, n}$ pointed stable (holomorphic) $k$-differentials. In particular, it means that $X$ is at worst nodal, $z_{1}, \ldots, z_{n}$ are distinct smooth points of $X$, and $\xi$ is a collection of $k$-differentials $\xi_{v}$ on irreducible components $X_{v}$ of $X$, which may have poles of order up to $k$ at the nodes of $X$. Furthermore, $\xi$ is required to satisfy the matching residue condition, namely, at any node $q \in X$ identifying $q_{1} \in X_{v_{1}}$ with $q_{2} \in X_{v_{2}}$, the equality $\operatorname{Res}_{q_{1}}^{k} \xi_{v_{1}}=(-1)^{k} \operatorname{Res}_{q_{2}}^{k} \xi_{v_{2}}$ holds (as discussed in Section 3.1, for $k$-differentials with poles of order at most $k$, the $k$-residue is equal to the coefficient of $z^{-k}(d z)^{k}$ in the Laurent expansion, and this equality for $k$-residues follows from the matching residue condition for $\omega_{X}$ at $q$ ).

Similarly to the case of meromorphic abelian differentials, for the strata of meromorphic $k$-differentials, we have to twist the ambient space by the polar part of the differentials. For this purpose, we write $\mu=\left(m_{1}, \ldots, m_{r}, m_{r+1}, \ldots, m_{r+s}, m_{r+s+1}, \ldots, m_{n}\right)$ with $m_{1}, \ldots, m_{r}>0$, 


\section{BAINBRIDGE ET AL.}

$m_{r+1}=\cdots=m_{r+s}=0$, and $m_{r+s+1}, \ldots, m_{n}<0$. Denote by $\tilde{\mu}=\left(m_{r+s+1}, \ldots, m_{n}\right)$ the polar part of $\mu$. Now, we define $\Omega^{k} \overline{\mathcal{M}}_{g, n}(\tilde{\mu}):=f_{*}\left(\omega_{f}^{k}\left(-\sum_{i=r+s+1}^{n} m_{i} Z_{i}\right)\right)$, where the $Z_{i}$ are the sections of the universal curve corresponding to the marked points. We call points $\left(X, \xi, z_{1}, \ldots, z_{n}\right) \in$ $\Omega^{k} \overline{\mathcal{M}}_{g, n}(\tilde{\mu})$ pointed stable $k$-differentials; this includes the holomorphic case when we set $\tilde{\mu}=\emptyset$.

Recall that the stratum $\Omega^{k} \mathcal{M}_{g}(\mu)$ parameterizes $\left(X, \xi, z_{1}, \ldots, z_{n}\right)$, where $X$ is a smooth genus $g$ curve, $z_{1}, \ldots, z_{n}$ are distinct marked points, and $\xi$ is a $k$-differential with zeros and poles at $z_{i}$ such that $\operatorname{ord}_{z_{i}} \xi=m_{i}$. It is clear that $\Omega^{k} \mathcal{M}_{g}(\mu)$ is contained in $\Omega^{k} \overline{\mathcal{M}}_{g, n}(\tilde{\mu})$. Note that there is a $\mathbb{C}^{*}$-action on $\Omega^{k} \overline{\mathcal{M}}_{g, n}(\tilde{\mu})$ by multiplication, which preserves the stratification of $\Omega^{k} \overline{\mathcal{M}}_{g, n}(\tilde{\mu})$. We denote the corresponding quotients by adding a letter $\mathbb{P}$. Now, we define the incidence variety compactification $\mathbb{P} \Omega^{k} \overline{\mathcal{M}}_{g, n}^{\text {inc }}(\mu)$ of the stratum $\Omega^{k} \mathcal{M}_{g}(\mu)$ to be the closure of the stratum in $\mathbb{P} \Omega^{k} \overline{\mathcal{M}}_{g, n}(\tilde{\mu})$. We remark that our notation implicitly means that the $n$ marked points are ordered. For the unordered version of the compactification, one can simply take the quotient by the respective symmetric group action.

As explained in [BCGGM18], the incidence variety compactification keeps both the scales of the limit differentials and the limit positions of zeros and poles, as $k$-differentials degenerate in a given stratum. Moreover, it determines the strata compactification in both the Deligne-Mumford space and in the Hodge bundle by forgetting the differentials and the markings, respectively. As can be seen by looking at $k$ th powers of abelian differentials, for which this is discussed in [BCGGM18], the incidence variety compactification records, in general, strictly more information than taking the strata compactification in either of the latter two spaces.

\subsection{Level graphs and twisted differentials}

In order to describe the incidence variety compactification of the strata of $k$-differentials, we need to introduce twisted $k$-differentials. The terminology we use here is closely related to that of [BCGGM18], with the extra complications coming from the particularities of $k$-residues.

Definition 3.2. Let $\mu=\left(m_{1}, \ldots, m_{n}\right)$ be a tuple of integers with $\sum_{i=1}^{n} m_{i}=k(2 g-2)$. A twisted $k$-differential $\eta$ of type $\mu$ on a stable $n$-pointed curve $\left(X, z_{1}, \ldots, z_{n}\right)$ is a collection of (possibly meromorphic) $k$-differentials $\eta_{v}$ on the irreducible components $X_{v}$ of $X$ such that no $\eta_{v}$ is identically zero and the following conditions hold:

(0) (Vanishing as prescribed) Each $k$-differential $\eta_{v}$ is holomorphic and non-zero outside the nodes and marked points of $X_{v}$. Moreover, if a marked point $z_{i}$ lies on $X_{v}$, then $\operatorname{ord}_{z_{i}} \eta_{v}=m_{i}$.

(1) (Matching orders) For any node of $X$ that identifies $q_{1} \in X_{v_{1}}$ with $q_{2} \in X_{v_{2}}$,

$$
\operatorname{ord}_{q_{1}} \eta_{v_{1}}+\operatorname{ord}_{q_{2}} \eta_{v_{2}}=-2 k \text {. }
$$

(2) (Matching residues at poles of order $k$ ) If at a node of $X$ that identifies $q_{1} \in X_{v_{1}}$ with $q_{2} \in X_{v_{2}}$, the condition $\operatorname{ord}_{q_{1}} \eta_{v_{1}}=\operatorname{ord}_{q_{2}} \eta_{v_{2}}=-k$ holds, then

$$
\operatorname{Res}_{q_{1}}^{k} \eta_{v_{1}}=(-1)^{k} \operatorname{Res}_{q_{2}}^{k} \eta_{v_{2}} .
$$

We next recall the notion of level graphs (see also [BCGGM18, Section 1.5]). Let $\Gamma$ be the dual graph of a nodal curve $X$. A full order on the irreducible components of $X$ is a relation $\succcurlyeq$ on the set $V$ of vertices of $\Gamma$ that is reflexive, transitive, and such that for any $v_{1}, v_{2} \in V$, at least one of the statements $v_{1} \succcurlyeq v_{2}$ and $v_{2} \succcurlyeq v_{1}$ holds. We remark that equality (denoted by $\left.\asymp\right)$ is permitted in our definition of a full order. Any map $\ell: V \rightarrow \mathbb{R}$ assigning real numbers to vertices of $\Gamma$ defines a full order on $\Gamma$ by setting $v_{1} \succcurlyeq v_{2}$ if and only if $\ell\left(v_{1}\right) \geqslant \ell\left(v_{2}\right)$. We call a dual graph $\Gamma$ equipped with a full order on its vertices a level graph and denote it by $\bar{\Gamma}$. Given a level 


\section{StRATA OF $k$-DIFFERENTIALS}

function $\ell$ and a real number $L$, we denote by $\bar{\Gamma}_{>L}$ (respectively, $\bar{\Gamma}_{\geqslant L}, \bar{\Gamma}_{=L}$, etc.) the subgraph of $\Gamma$ consisting of all vertices such that $\ell(v)>L$ (respectively, $\ell(v) \geqslant L, \ell(v)=L$, etc.) and all the edges connecting them.

In a level graph, an edge is called horizontal if it joins two vertices at the same level and called vertical otherwise. We also use the same names for the corresponding nodes of the curve. For a node $q$ joining two components $X_{v_{1}}$ and $X_{v_{2}}$ with $v_{1} \succcurlyeq v_{2}$, we denote by $q^{+}$and $q^{-}$the preimages of $q$ in $X_{v_{1}}$ and $X_{v_{2}}$, respectively. We also write $v^{+}(q)=v_{1}$ and $v^{-}(q)=v_{2}$ in this case. We will draw level graphs in such a way that the level function is given by projection to the vertical axis, so that the top level corresponds to the top of the pictured graph.

Along with the compatibility conditions (3) and (4-ab) in Definition 1.2 for twisted abelian differentials, we recall the main result of [BCGGM18].

TheOREM 3.3 ([BCGGM18]). A pointed stable abelian differential $\left(X, \omega, z_{1}, \ldots, z_{n}\right)$ is contained in the incidence variety compactification of a stratum $\mathbb{P} \Omega \mathcal{M}_{g}(\mu)$ if and only if the following conditions hold:

(i) There exists a level graph $\bar{\Gamma}$ such that its maxima are the irreducible components $X_{v}$ of $X$ on which $\omega$ is not identically zero.

(ii) There exists a twisted abelian differential $\eta$ of type $\mu$ on $X$, compatible with $\bar{\Gamma}$.

(iii) On every irreducible component $X_{v}$ where $\omega$ is not identically zero, $\eta_{v}=\left.\omega\right|_{X_{v}}$.

\section{Normalized covers and the global residue condition}

In this section, we prove Theorem 1.5, where the compatibility condition uses the $\widehat{g}$ lobal residue condition ( $\widehat{4}$ ), by reducing the problem to the case of abelian differentials (Theorem 3.3). This requires extending the notion of canonical covers, defined in Section 2 for smooth curves, to the case of nodal curves. More precisely, given a nodal curve $X$ with a twisted $k$-differential $\eta$, we want to construct a (possibly disconnected) admissible cover $\pi: \widehat{X} \rightarrow X$ such that $\pi^{*} \eta=\widehat{\omega}^{k}$, where $\widehat{\omega}$ is a twisted abelian differential on $\widehat{X}$. Here, the admissibility means that every node of $\widehat{X}$ maps to a node of $X$, every preimage of a node of $X$ is a node of $\widehat{X}$, and the ramification orders of $\pi$ at the two branches of any node of $\widehat{X}$ are equal (see [HM98, Section 3.G]). In addition, we want $\widehat{X}$ to carry a deck transformation $\tau$, cyclic of order $k$ as in the case of smooth canonical covers. Note that if $q$ is a node of $X$ identifying $q_{1} \in X_{v_{1}}$ with $q_{2} \in X_{v_{2}}$ and if $\eta$ is a twisted $k$-differential on $X$, then $q_{i}$ has $\operatorname{gcd}\left(k, \operatorname{ord}_{q_{i}} \eta_{i}\right)$ distinct preimages in the canonical cover of $X_{v_{i}}$. Thus by the matching orders condition for twisted $k$-differentials (condition (1) in Definition 3.2), $q_{1}$ and $q_{2}$ have the same number of preimages in the canonical covers $\widehat{X}_{v_{1}}$ and $\widehat{X}_{v_{2}}$. Hence, to construct $\widehat{X}$, we can pair preimages of $q_{1}$ with preimages of $q_{2}$ and identify the two points in each pair to form a node. In general there are many choices, but since $\widehat{X}$ is required to have a cyclic deck transformation $\tau$, we only need to identify one pair of the preimages of $q_{1}$ and $q_{2}$, and the remaining identifications are then determined from the induced actions of $\tau$ on $\widehat{X}_{v_{1}}$ and $\widehat{X}_{v_{2}}$.

To give the precise definition of the covers we want, fix a type $\mu=\left(m_{1}, \ldots, m_{n}\right)$ of $k$ differentials. We let $\widehat{m}_{i}:=\left(k+m_{i}\right) / \operatorname{gcd}\left(k, m_{i}\right)-1$ and let

$$
\widehat{\mu}:=(\underbrace{\widehat{m}_{1}, \ldots, \widehat{m}_{1}}_{\operatorname{gcd}\left(k, m_{1}\right)}, \underbrace{\widehat{m}_{2}, \ldots, \widehat{m}_{2}}_{\operatorname{gcd}\left(k, m_{2}\right)}, \ldots, \underbrace{\widehat{m}_{n}, \ldots, \widehat{m}_{n}}_{\operatorname{gcd}\left(k, m_{n}\right)}) .
$$

Given a cover $\pi: \widehat{X} \rightarrow X$ of a pointed stable curve $X$, we mark on $\widehat{X}$ all preimages of all the marked points in $X$. The order of the marked preimages in $\widehat{X}$ will not matter, except that we 


\section{BAINBRIDGE ET AL.}

list the preimages of the first marked point first, and so on.

Given a stable curve $X$ together with a twisted $k$-differential $\eta$, consider a triple

$$
(\pi: \widehat{X} \rightarrow X, \tau, \widehat{\omega})
$$

consisting of an admissible cover $\pi$ of degree $k$, its deck transformation $\tau$ of order $k$, and a twisted abelian differential $\widehat{\omega}$ on $\widehat{X}$ such that $\tau^{*} \widehat{\omega}=\zeta \widehat{\omega}$ and $\widehat{\omega}^{k}=\pi^{*} \eta$ on $\widehat{X}$, where $\zeta$ is the chosen primitive $k$ th root of unity. We will still call such triple a normalized cover (of a nodal curve) and note that its restriction to any irreducible component $X_{v}$ of $X$ is the normalized canonical cover as defined in Section 2.

We will often abbreviate the notation and say that $(\widehat{X}, \widehat{\omega})$ or $\widehat{X}$ is a normalized cover of $X$. Note that if such a normalized cover exists, then $\widehat{\mu}$ is the only type $\widehat{\omega}$ can possibly have, since the preimage of a marked point $z$ with $\operatorname{ord}_{z} \eta=m$ consists of $\operatorname{gcd}(k, m)$ distinct points at which $\widehat{\omega}$ has equal order. We now show that normalized covers always exist.

LEMma 4.1. For any twisted $k$-differential $\eta$ on a pointed stable curve $X$, there exists a normalized cover $(\widehat{X}, \tau, \widehat{\omega})$ such that $\widehat{\omega}$ is a twisted abelian differential of type $\widehat{\mu}$.

Proof. We first take normalized covers $\widehat{X}_{v} \rightarrow X_{v}$ of all irreducible components of $X$, with meromorphic abelian differentials $\widehat{\omega}_{v}$ on $\widehat{X}_{v}$. To construct $\widehat{X}$, it remains to identify the preimages of two branches of each node of $X$ in such a way that $\widehat{\omega}$ satisfies the conditions for a twisted abelian differential on $\widehat{X}$. By the construction of normalized covers for smooth curves, $\widehat{\omega}$ has prescribed vanishing, that is, it satisfies condition (0) in Definition 3.2 for twisted abelian differentials (that is, for $k=1$ ). To verify the matching orders condition (1), note that if $\operatorname{ord}_{q^{ \pm}} \eta=-k \pm m$ at a node $q$, then for any preimage $\widehat{q}_{i}$ of $q$,

$$
\operatorname{ord}_{\widehat{q}_{i}^{ \pm}} \widehat{\omega}=\frac{k+(-k \pm m)}{\operatorname{gcd}(k, m)}-1=\frac{ \pm m}{\operatorname{gcd}(k, m)}-1 ;
$$

hence, $\operatorname{ord}_{\widehat{q}_{i}^{+}} \widehat{\omega}+\operatorname{ord}_{\widehat{q_{i}}} \widehat{\omega}=-2$, and consequently condition (1) holds, independently of how the lifted branches of the nodes are identified.

If $q$ is a vertical node of $X$, then there is no matching residue condition imposed at its preimages, and the only restriction is that the curve would have an order $k$ automorphism $\tau$. Hence, one can choose any one preimage of $q^{+}$and identify it with any one preimage of $q^{-}$to form a node, and then the identifications of the rest of the preimages of $q^{ \pm}$are determined by the group action.

Finally, if $q_{1} \in X_{1}$ and $q_{2} \in X_{2}$ are identified to form a horizontal node $q \in X$, that is, if $\operatorname{ord}_{q_{1}} \eta_{1}=\operatorname{ord}_{q_{2}} \eta_{2}=-k$, then the matching residue condition (2) for twisted $k$-differentials requires $\operatorname{Res}_{q_{1}}^{k} \eta_{1}=(-1)^{k} \operatorname{Res}_{q_{2}}^{k} \eta_{2}$. Note that in this case, these $k$-residues are non-zero, as they are given by the coefficients of $z^{-k}(d z)^{k}$ in the local expression of $\eta$. The canonical covers of $X_{i}$ are then unramified over $q_{i}$, and the residues of $\widehat{\omega}$ at the preimages of $q_{i}$ are the $k$ th roots of $\operatorname{Res}_{q_{i}}^{k} \eta_{i}$, which differ from one another by multiplication by $k$ th roots of unity. Hence, for each preimage of $q_{1}$, there exists a unique preimage of $q_{2}$ where $\widehat{\omega}$ has opposite residue. Identifying these two preimages with opposite residues ensures that $\widehat{\omega}$ satisfies the matching residue condition (2) for twisted abelian differentials (and is the unique choice for such an identification).

Recall that every full order on a dual graph is induced by some level function. Let $\bar{\Gamma}$ be a full order on the dual graph $\Gamma$ of $X$ which is given by the level function $\ell: V(\Gamma) \rightarrow \mathbb{R}$. For any admissible cover $\pi: \widehat{X} \rightarrow X$, we define the lifted full order (or lifted level graph) $\widehat{\bar{\Gamma}}$ on the dual 


\section{StRATA OF $k$-DIFFERENTIALS}

graph $\widehat{\Gamma}$ of $\widehat{X}$ to be the full order given by the level function $\ell \circ \pi$ on $V(\widehat{\Gamma})$. It is easy to see that the lifted full order depends only on $\bar{\Gamma}$ and not on the choice of the level function.

Lemma 4.2. Let $\eta$ be a twisted $k$-differential on a pointed stable curve $X$ satisfying the partial order condition (3) for a level graph $\bar{\Gamma}$. Then for every normalized cover $(\pi: \widehat{X} \rightarrow X, \tau, \widehat{\omega})$, the twisted abelian differential $\widehat{\omega}$ also satisfies the partial order condition (3) for the lifted level graph $\widehat{\bar{\Gamma}}$.

Moreover, for any level $L$, the restriction of the normalized cover over $X_{=L}$ is uniquely determined by $\eta$, and the entire cover is further determined by choosing, for each vertical node $q$ of $\bar{\Gamma}$, one $\pi$-preimage of $q^{+}$and one $\pi$-preimage of $q^{-}$that are identified.

Proof. For any node $q$ of $X$, we have $\operatorname{ord}_{q^{ \pm}} \eta \geqslant-k$ if and only if $\operatorname{ord}_{\widehat{q}_{i}^{ \pm}} \widehat{\omega} \geqslant-1$ for any preimage $q_{i}$ of $q$. It follows that $\widehat{\omega}$ satisfies the partial order condition (3) for $\widehat{\bar{\Gamma}}$. The uniqueness of identifying preimages of a horizontal node on level $L$ and the way of identifying preimages of a vertical node were shown in the proof of Lemma 4.1 already.

Before proving Theorem 1.5, we remark that condition $(\widehat{4})$ depends on the choice of a normalized cover, as shown by the following example.

EXAMPLE 4.3 The global residue condition depends on the normalized cover. Let $X$ be a stable curve consisting of three irreducible components $X_{1}, X_{2}$, and $X_{3}$ such that $X_{1}$ meets $X_{2}$ at two nodes and $X_{2}$ meets $X_{3}$ at one node (see Figure 2). Let $\eta$ be a twisted quadratic differential (that is, $k=2$ ) such that $\eta_{1}$ and $\eta_{2}$ are squares of abelian differentials and $\eta_{3}$ is a primitive quadratic differential. Moreover, suppose that $\eta_{2}$ is the square of a meromorphic abelian differential that has poles at the nodes of $X_{1}$ without residue. Let us consider the two normalized covers $\widehat{X}^{(1)}$ and $\widehat{X}^{(2)}$ with lifted level graphs shown in Figure 2.
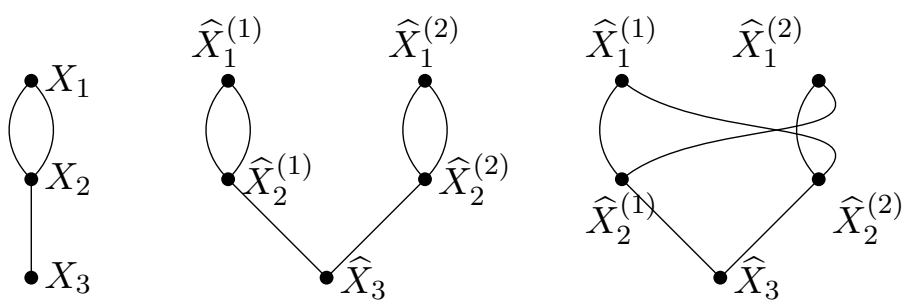

Figure 2. The level graphs $\bar{\Gamma}, \widehat{\bar{\Gamma}}_{1}$, and $\widehat{\bar{\Gamma}}_{2}$

The global residue condition $(\widehat{4})$ for $\widehat{\bar{\Gamma}}_{1}$ says that the residue of $\widehat{\omega}_{3}$ at each of the two nodes of $\widehat{X}_{3}$ is zero. Since the sum of these two residues is always zero by the residue theorem for $\widehat{\omega}_{3}$ on $\widehat{X}_{3}$, and the square of either residue of $\widehat{\omega}_{3}$ is equal to the 2-residue of $\eta_{3}$ at the node of $X_{3}$, it implies that condition ( $\widehat{4}$ ) for $\widehat{\bar{\Gamma}}_{1}$ is equivalent to imposing $\operatorname{Res}^{2} \eta_{3}=0$. On the other hand, the global residue condition $(\widehat{4})$ for $\widehat{\bar{\Gamma}}_{2}$ requires only that the sum of the two residues of $\widehat{\omega}_{3}$ at the two nodes of $\widehat{X}_{3}$ is equal to zero, which holds automatically by the residue theorem; the residues of $\widehat{\omega}_{3}$ for this normalized cover may be non-zero, differing from each other by a sign.

The rest of this section is devoted to the proof of Theorem 1.5. We first prove that the conditions are necessary. Then we develop an equivariant version of plumbing techniques used in [BCGGM18] to show that the conditions are also sufficient. 


\section{BAINBRIDGE ET AL.}

Proof of Theorem 1.5, the conditions are necessary. We need to show that if a pointed stable $k$-differential lies in the incidence variety compactification of a stratum, then it is associated with some twisted $k$-differential satisfying conditions (0)-(3) and $(\widehat{4})$. Except for condition $(\widehat{4})$, the argument is similar to the case of abelian differentials (see [BCGGM18] for more details). We start with a family of curves $f: \mathcal{X} \rightarrow \Delta$ over a disk that degenerates to the central fiber $X$, with a family $\xi_{t}$ of $k$-differentials of type $\mu$ for $t \in \Delta^{*}$, and let $Z_{i}$ be the sections of marked zeros and poles. Then for each irreducible component $X_{v}$ of $X$, there exists a scaling parameter $\ell_{v} \in \mathbb{Z}$ (in the sense of [BCGGM18, Lemma 4.1]) such that $\sum m_{i} Z_{i}$ is a section of the twisted relative $k$-dualizing sheaf $\omega_{f}^{k}\left(\sum \ell_{v} X_{v}\right)$. For later use, we make a degree $k$ base change such that $k \mid \ell_{v}$ for all $\ell_{v}$. Now the same proof as in the case $k=1$ leads to the necessary conditions (0), (1), and (2). Hence, we have constructed a twisted $k$-differential $(X, \eta)$, where $\eta_{v}:=\left.\lim _{t \rightarrow 0} t^{\ell_{v}} \xi_{t}\right|_{X_{v}}$. Moreover, the set of values $\left\{\ell_{v}\right\}$ defines a level graph $\bar{\Gamma}$, which ensures that the partial order condition (3) holds, again with the same proof as for $k=1$.

We now prove the necessity of condition $(\widehat{4})$. For $t \in \Delta^{*}$, let $\left(\pi_{t}: \widehat{X}_{t} \rightarrow X_{t}, \widehat{\omega}_{t}\right)$ be the family of normalized covers associated with the family of $k$-differentials $\xi_{t}$. It is well known that the Hurwitz space of connected admissible covers is proper (see [HM82, Section 4]). Since a branched cover of degree $d$ is connected if and only if the associated monodromy group in the symmetric group $S_{d}$ acts transitively on the $d$ sheets of the cover, and the transitivity condition was not really used in the argument of properness, the Hurwitz space of possibly disconnected admissible covers is also proper. ${ }^{1}$ It follows that the family $\pi_{t}$ extends over $t=0$ to some admissible cover $\pi: \widehat{X} \rightarrow X$, and the order $k$ deck transformation $\tau_{t}$ of $\pi_{t}$ extends to a deck transformation $\tau$ of order $k$ for the cover $\pi$ in the central fiber. Then the scaling parameters for the family $\widehat{X}_{t}$ of abelian differentials are equal to $\ell_{\pi\left(\widehat{X}_{v}\right)} / k$. This implies that the twisted abelian differential $\widehat{\omega}$ obtained as the scaling limit of $\widehat{\omega}_{t}$ is compatible with the lifted level graph $\widehat{\bar{\Gamma}}$. By the proof of necessity for abelian differentials given in [BCGGM18, Section 4.1], we conclude that $\widehat{\omega}$ satisfies the $\widehat{g}$ lobal residue condition imposed by $\widehat{\bar{\Gamma}}$. To show that $(\widehat{X}, \widehat{\omega}, \tau)$ is a normalized cover of $(X, \eta)$, the only remaining properties we need to justify are that $\tau^{*} \widehat{\omega}=\zeta \widehat{\omega}$ and that $\widehat{\omega}^{k}=\pi^{*} \eta$. It suffices to check them on each irreducible component $X_{v}$ of $X$. The first property follows from taking the limit of the equations

$$
\tau_{t}^{*}\left(t^{\ell_{v} / k} \widehat{\omega}_{t}\right)=t^{\ell_{v} / k} \tau_{t}^{*}\left(\widehat{\omega}_{t}\right)=t^{\ell_{v} / k} \zeta \widehat{\omega}_{t}=\zeta\left(t^{\ell_{v} / k} \widehat{\omega}_{t}\right)
$$

as $t$ goes to zero. The second property follows similarly from the fact that $\left(t^{\ell_{v} / k} \widehat{\omega}_{t}\right)^{k}=t^{\ell_{v}} \pi_{t}^{*}\left(\xi_{t}\right)$.

The proof of sufficiency proceeds by passing to normalized covers and applying the sufficiency for abelian differentials as in [BCGGM18]. The crucial ingredients of the proof for abelian differentials are the existence of a modification differential and the procedure of merging nearby zeros, which we now need to perform on an admissible cover in a way that is equivariant under the action of the automorphism $\tau$.

\footnotetext{
${ }^{1}$ Following a referee's suggestion, here is a more precise argument for the properness of the Hurwitz space of possibly disconnected admissible covers. Using the same notation, after a suitable base change for the family $\pi_{t}$, we may assume that the new family has no monodromy around $t=0$ in the sense that the total space has exactly as many connected components as any of the special fibers. Then we can treat each component separately and apply the usual properness of the connected case. In particular, these components all extend uniquely to $t=0$ after a possible further base change.
} 
LEMMA 4.4 (Existence of equivariant modification differentials). Suppose that $\widehat{Y}$ is a Riemann surface admitting an automorphism $\tau$ of order $\ell>1$. For any $\tau$-invariant collection $\mathcal{Q}$ of points in $\widehat{Y}$ and any function $r: \mathcal{Q} \rightarrow \mathbb{C}$ with $\tau^{*} r=\zeta r$, where $\zeta$ is a primitive $\ell$ th root of unity, there exists an abelian differential $\phi$ on $\widehat{Y}$ satisfying the following properties:

(i) The poles of $\phi$ are simple and contained in $\mathcal{Q}$.

(ii) The residue of $\phi$ at $q \in \mathcal{Q}$ is $r(q)$.

(iii) The equivariance $\tau^{*} \phi=\zeta \phi$ holds.

Proof. Decomposing $\mathcal{Q}$ into $\tau$-orbits and using that $\sum_{i=1}^{\ell} \zeta^{i}=0$ since $\ell>1$, we find that $\sum_{q \in \mathcal{Q}} r(q)=0$. Consequently, there is no restriction imposed by the residue theorem, and hence by the Mittag-Leffler theorem, there exists a meromorphic abelian differential $\phi_{0}$ on $\widehat{Y}$ satisfying properties (i) and (ii). To obtain the equivariance required in property (iii), we simply average while twisting by $\zeta$; that is, we let

$$
\phi:=\frac{1}{\ell} \sum_{i=0}^{\ell-1} \zeta^{-i}\left(\tau^{i}\right)^{*} \phi_{0} .
$$

Then $\phi$ satisfies properties (i) and (iii) by construction. Moreover, since $\tau^{*} r=\zeta r$, we have

$$
\operatorname{Res}_{q}\left(\zeta^{-1} \tau^{*} \phi_{0}\right)=\zeta^{-1} \operatorname{Res}_{\tau(q)} \phi_{0}=\zeta^{-1} \zeta \operatorname{Res}_{q} \phi_{0}
$$

which implies that $\operatorname{Res}_{q} \phi=\operatorname{Res}_{q} \phi_{0}=r(q)$. Hence $\phi$ also satisfies property (ii).

Proof of Theorem 1.5, the conditions are sufficient. Our goal is to show that given a twisted $k$ differential $\eta$ and a normalized cover $(\widehat{X}, \widehat{\omega})$ satisfying conditions $(0)-(3)$ and $(\widehat{4})$, there exists a family $f: \mathcal{X} \rightarrow \Delta$ of pointed stable curves such that for $t \neq 0$, the curve $X_{t}$ is smooth and carries a $k$-differential $\xi_{t}$ of type $\mu$ which converges to the pointed stable $k$-differential $\xi$ on $X$ associated with $\eta$. The proof will moreover show that $\eta$ is the scaling limit of $\xi$, in the sense of [BCGGM18, Lemma 4.1] and of the proof of necessity above.

By condition ( $\widehat{4}$ ) and the main result of [BCGGM18], the stable differential associated with the normalized cover $(\widehat{X}, \widehat{\omega})$ lies in the boundary of the incidence variety compactification for the stratum of abelian differentials of type $\widehat{\mu}$ (and if $\widehat{X}$ is disconnected, we then apply [BCGGM18] to each connected component of $\widehat{X}$ separately). Hence, there exists a family $\widehat{f}: \widehat{\mathcal{X}} \rightarrow \Delta$ of pointed stable curves such that for $t \neq 0$, the curve $\widehat{X}_{t}$ is smooth and carries an abelian differential $\widehat{\omega}_{t}$, while the scaling limit of $\widehat{\omega}_{t}$ is equal to $\widehat{\omega}$. We need to show that the construction of the family $\widehat{f}$ can be carried out in such a way that the deck transformation $\tau$ on the central fiber $\widehat{X}$ extends to an automorphism $\tilde{\tau}$ of $\widehat{\mathcal{X}}$ that, restricted to each fiber, is a deck transformation. Then the desired family $\mathcal{X}$ over $\Delta$ can be obtained by taking the quotient of $\widehat{X}$ under the extended automorphism $\tilde{\tau}$, and $\widehat{\omega}^{k}$ on $\widehat{X}$ will be the pullback of a twisted $k$-differential from $X$ that satisfies conditions $(0)-(3)$ and $(\widehat{4})$.

We recall the outline of the proof given in [BCGGM18] for abelian differentials and highlight the steps that need modification in the equivariant setting. The base of the induction, and in general the final cleanup step, is to smooth all horizontal nodes by classical plumbing. For this, we need to make sure that [BCGGM18, Proposition 4.4] applies equivariantly. Let $q$ be a horizontal node of $X$, and let $\widehat{q}$ be a preimage of $q$ on the normalized cover. The upshot is that the $\tau$-orbit of $\widehat{q}$ consists of $k$ distinct points, so that we can plumb one of them and then move it around using $\tau$. More precisely, having fixed a standard coordinate $u$ for abelian differentials provided in loc. cit., the plumbing (with fixture $\Omega_{t}=r(t) d u / u$ ) of the node $\widehat{q}$ depends only on the residue $r(t)$. 


\section{BAINBRIDGE ET AL.}

Hence, we choose this standard coordinate $u$ at $\widehat{q}$ and plumb there. Then at a node $\tau^{i}(\widehat{q}) \in \widehat{X}$, which is also a preimage of the node $q$, we use $u \circ \tau^{i}$ as the standard coordinate and apply $\tau^{i}$ to the previous plumbing construction, where $\tau$ acts on $\Omega$ by multiplication by $\zeta$. The resulting plumbing of $\widehat{X}$ is then $\tau$-equivariant by construction.

The induction step consists of joining a smoothing $f: \widehat{\mathcal{Y}} \rightarrow \Delta$ of the (possibly disconnected) subcurve $\widehat{X}_{>L}$ of components of level greater than $L$ to the subcurve $\widehat{X}_{=L}$. The differentials are scaled by the factors of $t^{\ell}$ for a level function $\ell: V(\widehat{\Gamma}) \rightarrow \mathbb{Z}_{\leqslant 0}$, which is inductively constructed such that the differences between the levels are sufficiently divisible. To apply higher-order plumbing [BCGGM18, Theorem 4.5], we need the existence of a modification differential $\phi$ (denoted by $\xi$ in loc. cit.) with the following properties. For every connected component $\widehat{Y}$ of $\widehat{X}_{>L}$, let $\left\{\widehat{q}_{i, j}\right\}$ denote the set of nodes of $\widehat{Y}$ that intersect $\widehat{X}_{=L}$, indexed in such a way that $\widehat{q}_{i, j}$ is a preimage of the node $q_{i}$ of $Y$, and such that $\tau\left(\widehat{q}_{i, j}\right)=\widehat{q}_{i, j+1}$, where the index $j$ is set to be modulo an appropriate divisor of $k$. Then the requirement on $\phi$ in loc. cit. is that $\operatorname{Res}_{\widehat{q}_{i, j}^{+}}(\phi)=-\operatorname{Res}_{\widehat{q}_{i, j}^{-}}(\widehat{\omega})$. Note that if $k \mid \operatorname{ord}_{q_{i}} \eta$, then the $\tau$-orbit of $\widehat{q}_{i, j}$ has cardinality $k$. If $k \nmid \operatorname{ord}_{q_{i}} \eta$, then Proposition 3.1 implies that $\operatorname{Res}_{\widehat{q}_{i, j}^{-}}(\widehat{\omega})=0$. Therefore, if the $\pi$-preimage of a connected component of $X_{>L}$ is a disjoint union of $k$ curves, we can directly apply [BCGGM18, Lemma 4.6] to obtain the desired modification differential $\phi$. On the other hand, if this preimage has $s<k$ connected components, we can use Lemma 4.4 with $\mathcal{Q}$ given by the set of nodes where $\operatorname{Res}_{\widehat{q}_{i, j}}(\widehat{\omega}) \neq 0$, with the function $r\left(\widehat{q}_{i, j}^{+}\right)=-\operatorname{Res}_{\widehat{q}_{i, j}^{-}}(\widehat{\omega})$, with $\ell=k / s$, and with $\tau^{s}$ as the automorphism on each connected component, in order to obtain the modification differential $\phi$.

The higher-order plumbing for the node $\widehat{q}_{i, j}$ is performed on some neighborhoods $\mathcal{D}^{ \pm}$of $\widehat{q}_{i, j}^{ \pm}$, where we plumb $\psi_{t}^{+}=t^{c}\left(\widehat{\omega}_{t}^{+}+t^{b} \phi_{t}\right)$ to $\psi_{t}^{-}=t^{b+c} \widehat{\omega}_{t}^{-}$using the plumbing fixture differential $\Omega_{t}$ given in [BCGGM18, Theorem 4.5]. Here, $b$ and $c$ are constants depending on ord $q_{i, j}^{+} \widehat{\omega}$ and on the level function. On the neighborhoods $\tau^{-1} \mathcal{D}^{ \pm}$of $\widehat{q}_{i, j-1}^{ \pm}$, consider the differentials $\tau^{*} \psi_{t}^{+}=\zeta \psi_{t}^{+}$ and $\tau^{*} \psi_{t}^{-}=\zeta t^{b+c} \widehat{\omega}_{t}^{-}$, where the equalities are implied by the $\tau$-equivariance of $\phi$. These can be glued using the plumbing fixture differential $\zeta \Omega_{t}$.

More precisely, we simplify the notation by writing $q$ for a node of $X$ and let $m=\operatorname{ord}_{q^{+}} \eta$. If $k \mid m$, the gluing of plumbing fixtures over $q$ can clearly be done as described above. Suppose $k \nmid m$, and let $(u, v)$ be local (standard) coordinates around the node $q$ such that $\eta=u^{m}(d u)^{k}$ and $\eta=v^{-m-2 k}(d v)^{k}$. Recall that there are $r=\operatorname{gcd}(m, k)$ preimages of the node $q$ and that the order $\widehat{m}$ of $\widehat{\omega}$ at the preimages is equal to $(m+k) / r-1$. Consider the families of degenerating cylinders $\left\{\left(t, u_{j}, v_{j}\right) \mid u_{j} v_{j}=t^{d}\right\}$ for some $d$, with indices $j=0, \ldots, r-1$, along with the forms $\Omega_{t}=t^{c} \zeta^{j} u_{j}^{\widehat{m}} d u_{j}$ and $\Omega_{t}=t^{b+c} \zeta^{j} v_{j}^{-\widehat{m}-2} d v_{j}$. Define the action $\tau^{\prime}\left(t, u_{j}, v_{j}\right)=\left(t, u_{j+1}, v_{j+1}\right)$ for $j=0, \ldots, r-2$ and $\tau^{\prime}\left(t, u_{r-1}, v_{r-1}\right)=\left(t, \zeta_{a}^{-r} u_{0}, \zeta_{a}^{r} v_{0}\right)$ for some $k$ th root of unity $\zeta_{a}$ to be determined. The induced action $\left(\tau^{\prime}\right)^{*}$ on $\Omega_{t}$ is clearly multiplication by $\zeta$ for $j \neq 0$. For $j=0$, the action on $\Omega_{t}$ is given by $\left(\tau^{\prime}\right)^{*}\left(t^{c} u_{0}^{\widehat{m}} d u_{0}\right)=\zeta_{a}^{m+k}\left(t^{c} u_{r-1}^{\widehat{m}} d u_{r-1}\right)$. In order to make it equal to $\zeta \Omega_{t}$ for $j=r-1$, we choose $\zeta_{a}$ such that $\zeta_{a}^{m+k}=\zeta^{r}$, which has a solution among the $k$ th roots of unity since $\operatorname{gcd}(m, k)=r$. Similarly, one can check it for the $v$-coordinates. Hence, the action $\tau^{\prime}$ extends $\tau$ on the families of degenerating cylinders. Consequently, the family $\widehat{f}_{\text {zeros }}$ of pointed stable differentials obtained by plumbing admits the desired automorphism $\tilde{\tau}$ that extends $\tau$.

The family of differentials $\widehat{f}_{\text {zeros }}$ does not yet have the right type $\widehat{\mu}$, because a multiple zero $\widehat{z}$ of $\widehat{\omega}_{t}$ may break into a collection of zeros, of the same total multiplicity, when a small modification differential $\phi$ is added. In the case of abelian differentials, for each zero $\widehat{z}$ of $\widehat{\omega}$ in the smooth locus of $\widehat{X}$, this issue is settled by merging zeros [BCGGM18, Lemma 4.7], which is a local operation 


\section{StRATA OF $k$-DIFFERENTIALS}

in a neighborhood $\mathcal{D}$ of the center of masses $\widehat{x}(t)$ of the zeros that $\widehat{z}$ splits to. It consists of replacing the family of differentials $\psi_{t}=t^{c}\left(\widehat{\omega}_{t}^{+}+t^{b} \phi_{t}\right)$ with another family of differentials $\psi_{t}^{\prime}$ with a unique zero of multiplicity equal to the sum of multiplicities of the dispersed zeros. As in the step of higher-order plumbing, we can perform this merging locally near one preimage $\widehat{z}$ of a dispersed zero, and then use the property $\tau^{*} \psi_{t}=\zeta \psi_{t}$ to replace, on $\tau^{-1}(\mathcal{D})$, the differential $\zeta \psi_{t}$ by $\zeta \psi_{t}^{\prime}$. This can be done, as in the previous paragraph, in such a way that $\tau^{*} \psi_{t}^{\prime}=\zeta \psi_{t}^{\prime}$. At the end, we obtain a family $\widehat{f}$ of pointed stable differentials of the correct type and still with an automorphism $\tilde{\tau}$ extending $\tau$ as desired.

\section{The global $k$-residue condition}

Throughout this section, we fix a nodal curve $X$ with a level graph $\bar{\Gamma}$ given by a level function $\ell$. Whenever we take normalized covers of $X$ or of its subcurves, we implicitly consider them endowed with a lifted full order provided by Lemma 4.2 .

Previously, we have characterized the incidence variety compactification of the strata of $k$ differentials in terms of the existence of a normalized cover satisfying the $\widehat{g}$ lobal residue condition $(\widehat{4})$. As Example 4.3 demonstrates, the choice of a normalized cover is fundamental in the global residue condition $(\widehat{4})$. In this section, we investigate the combinatorics of possible choices of a normalized cover. By going through the various possibilities, eventually we will establish the equivalence of the $\widehat{g}$ lobal residue condition $(\widehat{4})$ and the global $k$-residue condition (4) in Definition 1.4.

In order to establish the equivalence between conditions $(\widehat{4})$ and (4), the main tool we use is the following result.

Proposition 5.1. Let $(X, \eta)$ be a twisted $k$-differential. If the restriction $\left.\eta\right|_{Y}$ to a connected component $Y$ of $X_{>L}$ has a normalized cover $(\widehat{Y}, \tau, \widehat{\omega})$ with strictly fewer than $k$ connected components, then the global residue condition (4-ab) for abelian differentials in Definition 1.2 imposed by $\widehat{Y}$ on $\widehat{\omega}$ is automatically satisfied.

Proof. Let $\widehat{Y}_{1}$ be one of the $r<k$ connected components of $\widehat{Y}$. Denote by $q_{i}$ the nodes connecting $Y$ to $X_{=L}$, and let $\widehat{q}_{i, j}$ be the nodes connecting $\widehat{Y}_{1}$ to $\widehat{X}_{=L}$. Since the cover $\widehat{Y} \rightarrow Y$ is cyclic, the deck group must act transitively on the set of connected components of $\widehat{Y}$. It thus follows that $r$ divides $k$, and then that $\tau^{k / r}$ acts on $\widehat{Y}_{1}$, and since $\tau^{*}(\widehat{\omega})=\zeta \widehat{\omega}$, the sum of the residues of $\widehat{\omega}$ at the nodes $\widehat{q}_{i, j}$ is proportional to $\sum_{j=1}^{k / r} \zeta^{j r}$, which is zero because the total sum of $(k / r)$ th roots of unity is zero for $r<k$.

In order to apply the above proposition, we need to know when the preimage of a connected component of $X_{>L}$ can have fewer than $k$ connected components. The easiest case is described as follows.

Lemma 5.2. Suppose that the twisted $k$-differential $\eta_{v}$ on an irreducible component $X_{v}$ is not the $k$ th power of an abelian differential. For any lower level $L<\ell(v)$, let $Y$ be the connected component of $X_{>L}$ containing $X_{v}$. Then any normalized cover of $\left(Y,\left.\eta\right|_{Y}\right)$ has strictly fewer than $k$ connected components.

Proof. Let $(\widehat{Y}, \tau, \widehat{\omega})$ be a normalized cover of $\left(Y,\left.\eta\right|_{Y}\right)$. Then the restriction $\widehat{X}_{v} \rightarrow X_{v}$ of $\widehat{Y} \rightarrow Y$ is a canonical normalized cover of $\left(X_{v}, \eta_{v}\right)$. Since $X_{v}$ is not the $k$ th power of an abelian differential, $\widehat{X}_{v}$ has strictly fewer than $k$ connected components. Since, as above, the deck group of $\widehat{Y} \rightarrow Y$ acts 


\section{BAINBRIDGE ET AL.}

transitively on the set of connected components of $\widehat{Y}$, it follows that every connected component of $\widehat{Y}$ contains at least one connected component of $\widehat{X}_{v}$, and hence $\widehat{Y}$ also has strictly fewer than $k$ connected components.

As a result, we show that if either case i) or case ii) in the global $k$-residue condition (4) holds, then no extra residue condition is imposed.

Corollary 5.3. Suppose that a connected component $Y$ of $X_{>L}$ contains an irreducible component $X_{v}$ such that either $\eta_{v}$ is not the $k$ th power of an abelian differential, or $X_{v}$ contains a marked pole, that is, there exists a $z_{i} \in X_{v}$ with $m_{i}<0$. Then for any normalized cover of $(X, \eta)$, the global residue condition (4-ab) for abelian differentials imposed by $\widehat{Y}$ on $\widehat{\omega}$ is automatically satisfied.

Proof. If $\eta_{v}$ is not the $k$ th power of an abelian differential, then by Lemma 5.2, any normalized cover of $\left(Y,\left.\eta\right|_{Y}\right)$ has fewer than $k$ connected components. Hence, condition (4-ab) imposed by $\widehat{Y}$ is automatically satisfied by Proposition 5.1.

If $X_{v}$ contains a marked pole $z_{i}$ of order $m_{i}$, we distinguish between two cases. If $k \mid m_{i}$, then any abelian differential $\widehat{\omega}_{v}$ such that $\widehat{\omega}_{v}^{k}=\pi^{*} \eta_{v}$ has a pole of order $m_{i} / k$ at each of the $k$ marked preimages of $z_{i}$. Then each connected component of $\widehat{Y}$ contains a marked pole of the differential $\widehat{\omega}_{v}$, in which case there is no global residue condition (4-ab) imposed by that connected component. On the other hand if $k \nmid m_{i}$, then $\eta_{v}$ is not the $k$ th power of an abelian differential, and we are back to the beginning situation of the proof.

We now suppose that for every irreducible component $X_{v}$ of $Y$, the twisted $k$-differential $\eta_{v}$ is the $k$ th power of an abelian differential which is holomorphic away from the nodes. It remains to determine under what conditions such a connected component $Y$ of $X_{>L}$ imposes a non-

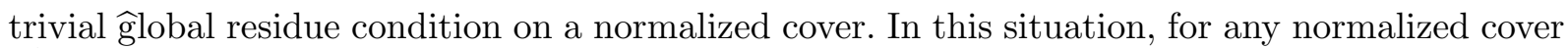
$(\widehat{Y}, \tau, \widehat{\omega})$ of $(Y, \eta)$, the preimage of any $X_{v}$ consists of $k$ isomorphic copies of $X_{v}$. We label these components by $\widehat{X}_{v}(0), \ldots, \widehat{X}_{v}(k-1)$ in such a way that $\tau$ sends $\widehat{X}_{v}(i)$ to $\widehat{X}_{v}(i+1)$ and $\widehat{X}_{v}(k-1)$ to $\widehat{X}_{v}(0)$. Then the normalized cover has the property that $\widehat{\omega}_{v}(i+1)=\zeta \widehat{\omega}_{v}(i)$, where $\zeta$ is the chosen primitive $k$ th root of unity and $\widehat{\omega}_{v}(i)=\left.\widehat{\omega}\right|_{\widehat{X}_{v}(i)}$.

We first consider the case of horizontal nodes. Let $X^{\prime}$ be a connected component of $X_{=K}$ for $K>L$. Note that there is a unique normalized cover $\left(\widehat{X}^{\prime}, \widehat{\omega}\right)$ of $\left(X^{\prime},\left.\eta\right|_{X^{\prime}}\right)$ which satisfies the matching residues condition (condition (2) of Definition 3.2) at the poles of order $k$, as the way to attach the preimages of the nodes is determined by this condition at the poles of order $k$. Hence, the fact that the normalized cover of $\left(X^{\prime},\left.\eta\right|_{X^{\prime}}\right)$ has fewer than $k$ components can be characterized in the following way.

LEMma 5.4. Let $X^{\prime}$ be a connected component of $X_{=K}$ such that for every irreducible component $X_{v}$ of $X^{\prime}$, the twisted $k$-differential $\eta_{v}$ is the $k$ th power of an abelian differential. Then the twisted $k$-differential $\left.\eta\right|_{X^{\prime}}$ is the $k$ th power of a twisted abelian differential if and only if the normalized cover $\widehat{X}^{\prime}$ has $k$ connected components.

In order to make this condition more clear, we discuss an example as follows.

EXAMPLE 5.5 (Horizontal criss-cross). Let $k=2$, and consider a twisted 2-differential obtained in the following way. We start from an abelian differential $(X, \omega)$, where $\omega$ has four simple poles $p_{1}, p_{2}, q_{1}$, and $q_{2}$. Suppose that the residues of $\omega$ at $p_{1}$ and $q_{1}$ are 1 and at $p_{2}$ and $q_{2}$ are -1 . We obtain a twisted 2-differential $(\bar{X}, \eta)$ by squaring $\omega$ and gluing $p_{1}$ with $q_{1}$ and $p_{2}$ with $q_{2}$. Clearly, 


\section{StRATA OF $k$-DIFFERENTIALS}

it satisfies condition (2) from Definition 3.2 at every node, as the 2-residues are all $( \pm 1)^{2}=1$. The canonical cover of $\left(X, \omega^{2}\right)$ has two components $X_{ \pm}$. In order to glue the nodes of these components to a normalized cover, we have no choice in the gluing but to pair the preimages $p_{i}^{+}$ of $p_{i}$ in $X_{+}$with $q_{i}^{-}$. Hence, the only possible normalized cover, represented in Figure 3 , has only one connected component. On the other hand, if the twisted 2-differential we started with had been obtained by identifying $p_{1}$ with $p_{2}$ and $q_{1}$ with $q_{2}$, then the normalized cover would have two connected components.
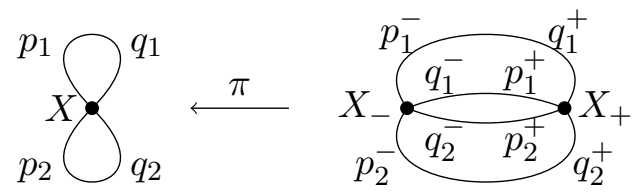

FiguRE 3. The dual graphs of $(\bar{X}, \eta)$ and its normalized cover

For vertical nodes, the construction of a normalized cover depends on identifying the preimages of the nodes, as described in the preceding section. If we choose the normalized cover $\widehat{Y} \rightarrow Y$ such that it consists of $k$ disjoint copies $\widehat{Y}(0), \ldots, \widehat{Y}(k-1)$ isomorphic to $Y$, then the global residue conditions imposed by these copies on the cover differ by multiplication by powers of $\zeta$. The residue condition imposed by $\widehat{Y}(0)$ is

$$
\sum_{q_{j} \in Y \cap X_{=L}} r_{q_{j}^{-}}=0
$$

for some numbers $r_{q_{j}^{-}}$such that $\left(r_{q_{j}^{-}}\right)^{k}=\operatorname{Res}_{q_{j}^{-}}^{k} \eta_{v^{-}\left(q_{j}\right)}$. Here, the particular choice of a $k$ th root $r_{q_{j}^{-}}$of the $k$-residue depends on which preimages of $q_{j}^{-}$on the normalized cover $\widehat{X}_{=L}$ are connected to the preimages of the nodes $q_{j}^{+}$lying on $\widehat{Y}(0)$. Since we can first choose these preimages arbitrarily and then identify preimages of $q_{j}^{+}$in the other copies $\widehat{Y}(i)$ with the preimages of $q_{j}^{-}$on $\widehat{X}_{=L}$ in a $\tau$-equivariant way, we have proven one implication of the following lemma for the construction of a normalized cover.

LEMMA 5.6. Let $Y$ be a connected component of $X_{>L}$. If there is a normalized cover $(\widehat{Y}, \widehat{\omega})$ of $(Y, \eta)$ satisfying the global residue condition (4-ab) which is the disjoint union of $k$ copies of $Y$, then there exists a normalized cover of $\left(Y \cup X_{=L}, \eta\right)$ which coincides with $(\widehat{Y}, \widehat{\omega})$ over $Y$ and satisfies the condition (4-ab) if and only if equation (5.1) holds for some $k$ th roots $r_{q_{j}^{-}}$of $k$-residues of $\eta$ at the nodes $q_{j} \in Y \cap X_{=L}$.

Proof. In the above discussion, we have verified the "only if" part. Conversely, suppose that equation (5.1) holds for some $r_{q_{j}^{-}}$. Then the residues of the $k$ th roots $\widehat{\omega}_{v}$ of $\eta_{v}$ on the canonical cover $\widehat{X}_{=L}$ at the nodes $\widehat{q}_{j, i}^{-}$lying over $q_{j}^{-}$are $\zeta^{i} r_{q_{j}^{-}}$for $i=0, \ldots, k-1$, if the nodes are numbered appropriately. In this numbering, we construct the normalized cover of $Y \cup X_{=L}$ by connecting the nodes $\widehat{q}_{j, i}$ to the component $\widehat{Y}(i)$ for all $j$. Equation (5.1) is then equivalent to the global residue condition imposed by $\widehat{Y}(0)$, and, more generally, $\widehat{Y}(i)$ imposes the $\tau^{i}$-image of this equation. We thus conclude that our gluing defines a normalized cover of $\eta$ over $Y \cup X_{=L}$ satisfying the condition (4-ab). 


\section{BAINBRIDGE ET AL.}

We can now conclude the case when a normalized cover contains a disjoint union of $k$ copies of $Y$.

Corollary 5.7. Let $Y$ be a connected component of $X_{>L}$. If the normalized cover $(\widehat{Y}, \widehat{\omega})$ of $(Y, \eta)$ is the disjoint union of $k$ copies of $Y$ and satisfies the condition (4-ab), then there exists a normalized cover over $Y \cup X_{=L}$ extending $\left.(\widehat{Y}, \widehat{\omega})\right)$ which satisfies the $\widehat{g}$ lobal residue condition imposed by $Y$ if and only if equation (1.4) for the $k$-residues is satisfied.

Proof. By definition of the polynomial $P_{n, k}$, equation (1.4) is simply the product of equations (5.1) for all possible choices of $k$ th roots of the relevant $k$-residues. Hence, one of those sums is zero if and only if their product is zero.

It remains to deal with the case when for every $K>L$ on every connected component $Y^{\prime}$ of $Y_{=K}$, the twisted $k$-differential $\left.\eta\right|_{Y^{\prime}}$ is the $k$ th power of a twisted abelian differential $\omega_{v}$, no $X_{v}$ contains a marked pole, but there exists a normalized cover $\widehat{Y}$ of $Y$ satisfying the global residue condition for abelian differentials (4-ab) that is not a disjoint union of $k$ copies of $Y$. Such a phenomenon occurs when we identify the irreducible components of $\widehat{Y}$ at the nodes in such a way that there is some crossing of the dual edges which cannot be straightened back (see $\widehat{\bar{\Gamma}}_{2}$ in Example 4.3). For this reason, we call such a construction a criss-cross.

Suppose that $Y$ has such a normalized cover $\widehat{Y}$, and then denote by $K$ the lowest level such that $\widehat{Y}$ restricted over $Y_{>K}$ consists of $k$ disjoint copies of $Y_{>K}$, while $\widehat{Y}$ restricted over some connected component $W$ of $Y_{\geqslant K}$ has fewer than $k$ connected components. By assumption, for each connected component $T_{\ell}$ of $W_{>K}$, the restriction of $\widehat{Y}$ over $T_{\ell}$ consists of $k$ disjoint copies $\widehat{T}_{\ell}(i)$ of $T_{\ell}$. Similarly, we denote by $U_{s}$ the connected components of $W_{=K}$. Since we suppose that the restriction $\left.\eta\right|_{U_{s}}$ is the $k$ th power of a twisted abelian differential, there are $k$ disjoint preimages of $U_{s}$ in the normalized cover (see Lemma 5.4). We denote by $\widehat{U}_{s}(i)$ these $k$ preimages. We set $\widehat{\omega}_{s}(i):=\zeta^{i} \omega_{s}$ for the restriction of $\pi^{*}(\eta)$ to $\widehat{U}_{s}(i)$. Moreover, denote by $\Gamma_{\text {red }}$ the level graph with two levels whose vertices are the connected components $T_{\ell}$ of $W_{>K}$ and $U_{s}$ of $W_{=K}$, and whose edges are the nodes between these components. Note that this graph has no horizontal edges. The graph $\widehat{\Gamma}_{\text {red }}$ is defined analogously by adding "hats" in the preceding definition.

Since, by hypothesis, $\widehat{W}$ has fewer than $k$ connected components, there exists a closed loop $\gamma$ in $\Gamma_{\text {red }}$ that has a lift $\widehat{\gamma}$ to $\widehat{\Gamma}_{\text {red }}$ which is not a closed loop (and then, by $\tau$-equivariance, it follows that no lift of $\gamma$ is a closed path). Given an edge $e$ of $\gamma$ connecting $T_{\ell}$ to $U_{s}$, its lift $\widehat{e}$ starting at $T_{\ell}(0)$ goes to some $U_{s}(o(e))$, and we set $\zeta_{e}:=\zeta^{o(e)}$. In the sum (5.1) for $Y=T_{\ell}$, the residue at the node $q_{j}$ corresponding to the edge $e_{j}$ is then given by $\zeta_{e_{j}} \operatorname{Res}_{q_{j}^{-}} \omega_{u}$. More generally, it follows from the $\tau$-equivariance that for a lift $\widehat{e}$ of $e$ connecting the component $\widehat{U}_{s}(a)$ to $\widehat{T}_{\ell}(b)$, we have $\zeta^{a-b}=\zeta_{e}$. Applying this observation recursively along $\gamma$, we see that the product $\prod_{e \in \gamma} \zeta_{e}^{ \pm 1}$ with alternating negative and positive exponents is a power of $\zeta$ whose exponent $i_{\gamma}$ gives the difference between the starting and the ending component of $\widehat{U}_{\ell_{1}}$ of any lift $\widehat{\gamma}$ of $\gamma$. By the hypothesis that $\widehat{\gamma}$ is not closed, this exponent $i_{\gamma}$ is not zero modulo $k$, and hence the product of the roots with alternating exponents \pm 1 in the above is not equal to 1 .

Now for the converse, suppose that there exists a connected component $W$ of $X_{\geqslant K}$ such that equation (5.1) is satisfied by taking $Y=T_{\ell}$, where the $T_{\ell}$ are the connected components of $W_{>K}$. Suppose, moreover, that there exists a loop $\gamma \in \Gamma_{\text {red }}$ such that the alternating product $\prod_{e \in \gamma} \zeta_{e}^{ \pm 1}$ is not equal to 1 . Then, as explained in Lemma 5.6, we can construct a normalized cover of $\left(W,\left.\eta\right|_{W}\right)$ satisfying condition (4-ab) by identifying the preimages of the nodes between the components $T_{\ell}$ and $W_{=K}$ in the way given by equation (5.1). As explained in the previous 


\section{StRATA OF $k$-DIFFERENTIALS}

paragraph, the assumption that the alternating product of the roots along $\gamma$ is not 1 implies that the lifts of $\gamma$ in $\widehat{\Gamma}_{\text {red }}$ are not closed paths. Hence, there is a normalized cover with fewer than $k$ connected components above $W$, and Proposition 5.1 can be applied.

To conclude this discussion, we would like to state the condition for the loop $\gamma$ in the dual graph of $W$ directly (instead of in $\Gamma_{\text {red }}$ ). This can be done as follows. With every path in the dual graph of $W$, we associate a path in $\Gamma_{\text {red }}$ by contracting the edges between vertices of the connected components $T_{\ell}$ of $W_{>K}$. Conversely, with a path in $\Gamma_{\text {red }}$, we associate a (not necessarily unique) path in the dual graph of $W$ such that it has the same edges joining the connected components $T_{\ell}$ of $W_{>K}$ to the connected components $U_{s}$ of $W_{=K}$ and then connects arbitrarily within each $T_{\ell}$. Clearly, the condition on $\gamma$ can be reformulated by saying that there exists a loop $\gamma$ in the dual graph of $W$ such that the alternating product of the roots associated with the edges of $\gamma$ touching $W_{=K}$ is not equal to 1 .

Summarizing the above discussion, we have proved the following proposition.

Proposition 5.8. Let $(Y, \eta)$ be a twisted $k$-differential satisfying condition (3) such that for every $K>L$ on every connected component $Y^{\prime}$ of $Y_{=K}$, the twisted $k$-differential $\left.\eta\right|_{Y^{\prime}}$ is the $k$ th power of a twisted abelian differential $\omega_{v}$ without marked poles. Then there exists a normalized cover of $(Y, \eta)$ satisfying condition (4-ab) which is not a disjoint union of $k$ copies of $Y$ if and only if there exist a level $K$, a connected component $W$ of $Y_{\geqslant K}$, and a simple closed path $\gamma$ in the dual graph of $W$ such that for every connected component $T_{\ell}$ of $W_{>K}$ and every edge $e$ in the set $E_{\ell}$ of nodes connecting $T_{\ell}$ to $Y_{=K}$, there exists a $k$ th root of unity $\zeta_{e}$ satisfying

$$
\sum_{e \in E_{\ell}} \zeta_{e} \operatorname{Res}_{q_{e}^{-}} \omega_{v^{-}(e)}=0 \quad \text { with } \prod_{e \in \gamma \cap E} \zeta_{e}^{ \pm 1} \neq 1,
$$

where $E=\bigcup_{\ell} E_{\ell}$ and the last product has alternating \pm 1 exponents along $\gamma$.

We are now ready to prove that the global residue condition $(\widehat{4})$ is equivalent to the global $k$-residue condition (4).

Proof of the equivalence of $(\widehat{4})$ and (4). We assemble the statements proven above to show that, for a twisted $k$-differential, satisfying conditions $(0)-(4)$ is equivalent to the existence of a normalized cover satisfying condition (4-ab).

Suppose first that the global $k$-residue condition (4) holds. We will construct the normalized cover satisfying condition $(\widehat{4})$ inductively on the number of levels. The claim is empty, hence true, for twisted $k$-differential with one level. Suppose that the claim is true for every twisted $k$-differential with at most $|L|$ levels satisfying condition (3) (we prefer using negative levels, so that the maximum is at level 0 , so $L$ is negative). Let $(X, \eta)$ be a twisted $k$-differential with $|L|+1$ levels satisfying conditions (3) and (4), where the levels are $0,-1, \ldots, L$ for simplicity. Let $Y_{1}, \ldots, Y_{r}$ be the connected components of $X_{>L}$. By assumption, for every $Y_{i}$ there exists a normalized cover $\widehat{Y}_{i}$. If condition iii) holds for $Y_{i}$, then $\widehat{Y}_{i}$ has fewer than $k$ connected components according to Lemma 5.4. Moreover, if condition iv) holds for $Y_{i}$, we may assume that $\widehat{Y}_{i}$ has fewer than $k$ connected components, since equations (1.3) and (1.2) are exactly the hypothesis of Proposition 5.8.

Suppose that one of the first four conditions i), ii), iii), and iv) in (4) holds for $Y_{i}$. Then by Corollary 5.3 (for conditions i) and ii)) and Proposition 5.1 (for conditions iii) and iv)), any normalized cover of $(X, \eta)$ will satisfy the global residue condition for abelian differentials imposed by $\widehat{Y}_{i}$. Now if none of these conditions holds for $\widehat{Y}_{i}$, then condition v) must be satisfied. We use, at $Y_{i}$, a normalized cover given in Corollary 5.7, which satisfies the global residue condition 


\section{BAINBRIDGE ET AL.}

for abelian differentials at every preimage of every component $Y_{i}$. Hence, we have constructed a normalized cover of $X$ satisfying condition (4-ab), proving that condition $(\widehat{4})$ holds for $(X, \eta)$. Indeed, we get a little more. If every component $Y_{i}$ has $k$ disjoint preimages and if the level $K$ in Proposition 5.8 coincides with $L$, then there exists a normalized cover of $X$ satisfying (4-ab) with fewer than $k$ connected components.

Conversely, suppose that the global residue condition $(\widehat{4})$ holds. We will show inductively on the number of levels that condition (4) holds. If there is only one level, the claim is again empty, hence true. Suppose that the claim is true for every twisted $k$-differential with at most $|L|$ levels satisfying condition $(3)$. Let $(X, \eta)$ be a twisted $k$-differential with $|L|+1$ levels satisfying conditions $(3)$ and $(\widehat{4})$. We denote by $(\widehat{X}, \widehat{\omega})$ a normalized cover of $(X, \eta)$ given by condition $(\widehat{4})$. Now by induction, condition (4) holds for every level $K>L$ and for every connected component of $X_{>K}$. Let $Y_{1}, \ldots, Y_{r}$ be the connected components of $X_{>L}$. For each $Y_{i}$, we will analyze the global residue condition for abelian differentials (4-ab) induced by $\widehat{Y}_{i}$ on $\widehat{\omega}$.

First, the global residue condition can be void if the preimages $\widehat{Y}_{i}$ of $Y_{i}$ contain a marked pole. Then the component $Y_{i}$ also contains a marked pole, and hence condition i) in (4) is satisfied. Next, the global residue condition for abelian differentials can be automatically satisfied if there are fewer than $k$ connected preimages of $Y_{i}$ in $\widehat{X}$. This can happen either because the restriction of $\eta$ on $Y_{i}$ is not the $k$ th power of an abelian differential or because there is some criss-cross gluing of nodes in $\widehat{X}$ over $Y_{i}$. In the former, condition ii) is satisfied. In the latter, either the criss-cross occurs with horizontal nodes and the hypothesis of Lemma 5.4 is satisfied, leading to condition iii). Or the criss-cross occurs with vertical nodes and the hypothesis of Proposition 5.8 must be satisfied for some level $K>L$, leading to condition iv). If none of these cases happens, then the global residue condition for abelian differentials induced by $\widehat{Y}_{i}$ is given by the vanishing of a factor of the polynomial defined in equation (1.1), which gives equation (1.4) of condition (4). Hence, condition (4) holds at level $L$.

\section{Dimension of spaces of twisted $k$-differentials}

In this section, we prove Theorem 1.6. Let $\bar{\Gamma}$ be a level graph and $h$ be the number of horizontal edges of $\bar{\Gamma}$. Recall that $\Omega^{k, \text { ab }} \mathcal{M}_{g}(\mu)$ is the union of the components of $\Omega^{k} \mathcal{M}_{g}(\mu)$ consisting of $k$ th powers of holomorphic abelian differentials, and $\Omega^{k \text {,non-ab }} \mathcal{M}_{g}(\mu)$ is the union of all the other components. We denote by $\mathfrak{W}^{k, \mathrm{ab}}(\bar{\Gamma})$ (respectively, $\mathfrak{W}^{k, \text { non-ab }}(\bar{\Gamma})$ ) the space of twisted $k$-differentials of type $\mu$ compatible with $\bar{\Gamma}$ which can be smoothed into $\Omega^{k, \text { ab }} \mathcal{M}_{g}(\mu)$ (respectively, $\Omega^{k \text {,non-ab }} \mathcal{M}_{g}(\mu)$ ); that is, the corresponding twisted $k$-differential is a scaling limit of a one-parameter family in $\Omega^{k, \text { ab }} \mathcal{M}_{g}(\mu)$ (respectively, $\Omega^{k, \text { non-ab }} \mathcal{M}_{g}(\mu)$ ). We emphasize that the underlying dual graph of every point in $\mathfrak{W}^{k, \mathrm{ab}}(\bar{\Gamma})$ (respectively, $\mathfrak{W}^{k, \text { non-ab }}(\bar{\Gamma})$ ) is exactly $\Gamma$, and we allow global scaling of the differentials on the components $X_{v}$ corresponding to the vertices $v$ of $\Gamma$. For $k=1$, we simply use $\mathfrak{W}^{\mathrm{ab}}(\bar{\Gamma})$ to denote the space of twisted abelian differentials of type $\mu$ compatible with $\bar{\Gamma}$, which in the above notation is either $\mathfrak{M}^{1, \mathrm{ab}}(\bar{\Gamma})$ if $\mu$ is a holomorphic signature or $\mathfrak{M}^{1, \text { non-ab }}(\bar{\Gamma})$ otherwise. We will verify the dimension count in Theorem 1.6 in the following two steps, first for $k=1$ and then for $k \geqslant 2$.

TheOREM 6.1. The space $\mathfrak{W}^{\mathrm{ab}}(\bar{\Gamma})$ of twisted abelian differentials either is empty or has dimension equal to $\operatorname{dim} \Omega \mathcal{M}_{g}(\mu)-h$.

TheOREM 6.2. For $k \geqslant 2$, the space $\mathfrak{W}^{k, \text { non-ab }}(\bar{\Gamma})$ either is empty or has dimension equal to $\operatorname{dim} \Omega^{k, \text { non-ab }} \mathcal{M}_{g}(\mu)-h$. 


\section{StRATA OF $k$-DIFFERENTIALS}

The same result holds when we replace "non-ab" with "ab" in Theorem 6.2; its proof simply reduces to the abelian case as in Theorem 6.1 , because any deformation of a $d$ th power of a $(k / d)$ differential remains a $d$ th power, as explained in and after Theorem 2.1.

We do some preparations before proving these theorems. First, we can reduce the situation to $h=0$, that is, $\bar{\Gamma}$ has no horizontal edges, since such a node is locally smoothable by classical plumbing, which affects the dimension count precisely by one. Hence, from now on, we assume that the level graph $\bar{\Gamma}$ has the property that $h=0$.

Consider the case of abelian differentials. Take a pointed topological surface $\Sigma$ with $\Lambda \subset \Sigma$ as a disjoint union of simple closed curves such that the degenerate surface with dual graph $\Gamma$ is obtained by pinching curves in $\Lambda$ to the corresponding nodes. Denote by $\Lambda^{\circ}$ an open thickening of $\Lambda$ with upper boundary $\Lambda^{+}$and lower boundary $\Lambda^{-}$. Let $P \subset \Sigma \backslash \Lambda^{\circ}$ be the set of marked points corresponding to the interior poles of the differentials and $Z \subset \Sigma \backslash \Lambda^{\circ}$ the set of those corresponding to the interior zeros.

The compatibility of twisted abelian differentials with $\bar{\Gamma}$ is governed by the global residue condition (4-ab), which is imposed to the residue assignments at the nodes of the degenerate surface. Moreover, such residue assignments should also satisfy the residue theorem on each irreducible component of the surface. Therefore, we define the subspaces of residue assignments

$$
R^{\text {grc }} \subseteq R^{\text {res }} \subseteq \mathbb{C}^{h^{0}(\Lambda)},
$$

where $R^{\text {res }}$ is the subspace satisfying the residue theorem at the irreducible components of $\Sigma \backslash \Lambda$ that have no marked poles in the interior but have poles at some resulting nodes (that is, those lower-level components without poles in the interior that are moreover not local maxima of $\bar{\Gamma}$ ), and where $R^{\text {grc }}$ is the subspace cut out further by the global residue condition for twisted differentials compatible with $\bar{\Gamma}$. Let $c_{\mathrm{L}}$ be the number of irreducible lower-level components without poles in the interior that are not local maxima of $\bar{\Gamma}$. Note that each component counted in $c_{\mathrm{L}}$ admits an independent condition for the constraint of the residue theorem, since every (polar) node belongs to a unique such component. It follows that

$$
h^{0}(\Lambda)-\operatorname{dim} R^{\text {res }}=c_{\mathrm{L}} .
$$

Also note that in our setting, the residue map

$$
\mathfrak{W}^{\mathrm{ab}}(\bar{\Gamma}) \rightarrow R^{\mathrm{res}}
$$

factors through $R^{\text {grc }}$.

Next, we give an alternative form of the global residue condition (4-ab). Without loss of generality, assume that the $N$ levels of $\bar{\Gamma}$ are labeled by zero for the top level and by $-i$ for the $i$ th level for $1 \leqslant i \leqslant N-1$. Treating $\Lambda$ as the set of edges of $\bar{\Gamma}$, define a subset $\Lambda_{j} \subseteq \Lambda$ by

$$
\Lambda_{j}=\left\langle\lambda \in \Lambda: \ell\left(v^{-}(\lambda)\right) \leqslant-j\right\rangle .
$$

Namely, $\Lambda_{j}$ is the subset of simple closed curves in $\Lambda$ whose corresponding edges in $\bar{\Gamma}$ have lower ends on level at most $-j$. Let $V_{j} \subset H_{1}(\Sigma \backslash P, Z)$ be the image subgroup generated by the classes of the simple closed curves in $\Lambda_{j}$ under the map $H_{1}(\Lambda) \rightarrow H_{1}(\Sigma \backslash P, Z)$ induced by the inclusion $\Lambda \hookrightarrow \Sigma$. We thus obtain a filtration of linear subspaces

$$
0=V_{N} \subseteq V_{N-1} \subseteq \cdots \subseteq V_{1}
$$

Since any residue assignment to the nodes of the pinched surface corresponds to a function $\rho: \Lambda \rightarrow \mathbb{C}$, we can characterize the subspace $R^{\text {grc }}$ as follows. 


\section{BAINBRIDGE ET AL.}

Proposition 6.3. A residue assignment $\rho: \Lambda \rightarrow \mathbb{C}$ satisfies the global residue condition if and only if there exist linear maps

$$
\rho_{j}: V_{j} / V_{j+1} \rightarrow \mathbb{C}, \quad \text { for } j=1, \ldots, N-1,
$$

such that $\rho_{j}(\lambda)=\rho(\lambda)$ for all simple closed curves $\lambda$ in $\Lambda$, where $j$ is determined by $\ell\left(v^{-}(\lambda)\right)=-j$.

In particular, $\operatorname{dim} R^{\mathrm{grc}}=\operatorname{dim} V_{1}=\operatorname{dim} \operatorname{Im}\left(H_{1}(\Lambda) \rightarrow H_{1}(\Sigma \backslash P, Z)\right)$.

Proof. Given the collection of $\rho_{j}$, for every $j$ and every connected component $Y$ of $\bar{\Gamma}_{>-j}$ without marked poles in the interior, the sum of the residues on all (down-going) edges of $Y$ is zero, as one can see by summing the corresponding boundary cycles in $H_{1}(\Sigma \backslash P, Z)$, and the edges to levels below $-j$ contribute zero since $\rho_{j}$ maps $V_{j+1}$ to zero. This zero summation is precisely the global residue condition (4-ab). The converse is just a reformulation of the global residue condition.

The second statement about $\operatorname{dim} R^{\operatorname{grc}}$ follows from the first, since $\rho$ is uniquely specified by any collection of $\rho_{j} \in\left(V_{j} / V_{j+1}\right)^{*}$ and $\sum_{j=1}^{N-1} \operatorname{dim}\left(V_{j} / V_{j+1}\right)=\operatorname{dim} V_{1}$.

Proof of Theorem 6.1. The stratum $\Omega \mathcal{M}_{g}(\mu)$ is locally modeled on the periods of the relative homology group $H_{1}(\Sigma \backslash P, Z)$. The space of twisted abelian differentials compatible with $\bar{\Gamma}$ except for the global residue condition is locally modeled on the periods of the homology group $H_{1}\left(\Sigma \backslash\left\{P \cup \Lambda^{\circ}\right\}, \Lambda^{+} \cup Z\right)$ by applying Corollary 2.3 to all components of $\Sigma \backslash \Lambda^{\circ}$ separately. Since the residue map from this space of twisted differentials to $R^{\text {res }}$ is dominant and equidimensional over its image (being the composition of a local biholomorphism and a linear projection under period coordinates), it suffices to show that

$$
h_{1}\left(\Sigma \backslash\left\{P \cup \Lambda^{\circ}\right\}, \Lambda^{+} \cup Z\right)-h_{1}(\Sigma \backslash P, Z)=\operatorname{dim} R^{\text {res }}-\operatorname{dim} R^{\text {grc }} .
$$

Take the long exact sequence of homology of the triple $Z \subset \Lambda \cup Z \subset \Sigma \backslash P$

$$
\begin{gathered}
\stackrel{\left.H_{2}(\Lambda \cup Z, Z)=0 \longrightarrow H_{2}(\Sigma \backslash P, Z) \longrightarrow H_{2}(\Sigma \backslash P, \Lambda \cup Z)\right)}{\left.\hookrightarrow H_{1}(\Lambda \cup Z, Z) \longrightarrow H_{1}(\Sigma \backslash P, Z) \longrightarrow H_{1}(\Sigma \backslash P, \Lambda \cup Z)\right)} \\
\hookrightarrow H_{0}(\Lambda \cup Z, Z) \longrightarrow H_{0}(\Sigma \backslash P, Z)=0 .
\end{gathered}
$$

Let $c:=h_{2}(\Sigma \backslash P, \Lambda \cup Z)$, which equals $h^{0}(\Sigma \backslash\{\Lambda \cup Z\}, P)$ by duality (see [Spa66, Theorem 6.2.17]), which is the number of connected components of $\Sigma \backslash \Lambda$ without poles in the interior. Let $\delta_{P}:=$ $h_{2}(\Sigma \backslash P, Z)=h^{0}(\Sigma \backslash Z, P)$, which is 1 if $P=\emptyset$ and is 0 otherwise. In addition, note that $h_{i}(\Lambda \cup Z, Z)=h_{i}(\Lambda)$ for $i=0,1$ and the same for their cohomology, all of which are equal to the number of simple closed curves in $\Lambda$, that is, the number of edges of $\bar{\Gamma}$. Also note that by applying the excision theorem to $\Lambda^{\circ}$, we have

$$
h_{i}\left(\Sigma \backslash\left\{P \cup \Lambda^{\circ}\right\}, \Lambda^{+} \cup \Lambda^{-} \cup Z\right)=h_{i}(\Sigma \backslash P, \Lambda \cup Z) .
$$

Using these observations, we can deduce from the long exact sequence that

$$
h_{1}\left(\Sigma \backslash\left\{P \cup \Lambda^{\circ}\right\}, \Lambda^{+} \cup \Lambda^{-} \cup Z\right)-h_{1}(\Sigma \backslash P, Z)=c-\delta_{P} .
$$

In addition, the exact sequence and Proposition 6.3 imply that

$$
\operatorname{dim} R^{\mathrm{grc}}=h_{1}(\Lambda \cup Z, Z)-\left(c-\delta_{P}\right)=h^{0}(\Lambda)-\left(c-\delta_{P}\right) .
$$

Note that (6.3) implies that $c=h_{2}\left(\Sigma \backslash\left\{P \cup \Lambda^{\circ}\right\}, \Lambda^{+} \cup \Lambda^{-} \cup Z\right)$. Combining this with the long exact sequence of homology of the triple $\Lambda^{+} \cup Z \subset \Lambda^{+} \cup \Lambda^{-} \cup Z \subset \Sigma \backslash\left\{P \cup \Lambda^{\circ}\right\}$, we obtain that

$$
h_{1}\left(\Sigma \backslash\left\{P \cup \Lambda^{\circ}\right\}, \Lambda^{+} \cup \Lambda^{-} \cup Z\right)-h_{1}\left(\Sigma \backslash\left\{P \cup \Lambda^{\circ}\right\}, \Lambda^{+} \cup Z\right)=c-c_{\mathrm{M}},
$$




\section{STRATA OF $k$-DIFFERENTIALS}

where $c_{\mathrm{M}}:=h_{2}\left(\Sigma \backslash\left\{P \cup \Lambda^{\circ}\right\}, \Lambda^{+} \cup Z\right)=h^{0}\left(\Sigma \backslash\left\{Z \cup \Lambda^{\circ}\right\}, \Lambda^{-} \cup P\right)$ is the number of connected components of $\Sigma \backslash \Lambda$ containing no poles, either in the interior or at the nodes obtained by pinching the (lower boundary) components of $\Lambda$. Said differently, $c_{\mathrm{M}}$ is the number of pole-free local maxima of $\bar{\Gamma}$.

Using the obvious relation $c=c_{\mathrm{M}}+c_{\mathrm{L}}$, combining (6.4) with (6.6), and comparing this to the difference of (6.5) and (6.1), we thus obtain the desired (6.2).

As a consequence of the above calculation, we also obtain that

$$
\operatorname{dim} R^{\mathrm{res}}-\operatorname{dim} R^{\mathrm{grc}}=c_{\mathrm{M}}-\delta_{P} .
$$

Namely, the number of independent global residue conditions is precisely the number of pole-free local maxima of $\bar{\Gamma}$, up to one global redundancy if the set of marked poles $P$ is empty.

Now we prove the case for $k \geqslant 2$, which is essentially an equivariant version of the preceding proof.

Proof of Theorem 6.2. We first give the setting which allows us to model the stratum. Fix a twisted $k$-differential $(X, \eta)$ and a connected normalized cover $(\widehat{X}, \widehat{\omega}, \pi)$ satisfying the global residue condition for abelian differentials (4-ab). The assumption that $\widehat{X}$ is connected is not a restriction, since otherwise we consider $(k / d)$-differentials, where $d$ is the number of connected components of $\widehat{X}$, and any deformation of a $d$ th power of a $(k / d)$-differential remains a $d$ th power. Take two topological surfaces $\widehat{\Sigma}$ and $\Sigma$ with two disjoint unions of simple closed curves $\widehat{\Lambda} \subset \widehat{\Sigma}$ and $\Lambda \subset \Sigma$ such that $\widehat{X}$ and $X$ are obtained by pinching curves in $\widehat{\Lambda}$ and $\Lambda$, respectively, and such that $\pi$ lifts to a cover from $\widehat{\Sigma}$ to $\Sigma$. Denote by $P$ the set of poles of $\eta$ with pole order at least $k$ in the interior of $\Sigma \backslash \Lambda$. Denote by $Z$ the set of zeros along with the remaining poles in the interior of $\Sigma \backslash \Lambda$. We similarly denote by $\widehat{P}$ and $\widehat{Z}$ the sets of preimages under $\pi$ of $P$ and $Z$ in the interior of $\widehat{\Sigma} \backslash \widehat{\Lambda}$, which correspond to the interior poles and zeros of the lifted twisted differential $\widehat{\omega}$, respectively.

The deck transformation $\tau$ on $\widehat{X}$ induces a deck transformation on $\widehat{\Sigma}$, which we still denote by $\tau$. We denote by $E_{i}$ the eigenspaces of various homology groups where $\tau$ acts by the eigenvalue $\zeta$ (the chosen primitive $k$ th root of unity) and denote the dimension of $E_{i}$ by $e_{i}$. Similarly, we use $E^{i}$ and $e^{i}$ for the $\zeta$-eigenspaces of cohomology groups and their dimensions. Let $\Sigma_{k}$ be the set of connected components of $\Sigma \backslash \Lambda$ where the twisted $k$-differential $\eta$ is the $k$ th power of an abelian differential, and let $\Lambda_{k} \subset \Lambda$ be the subset of curves where the vanishing orders of $\eta$ at the corresponding nodes are a multiple of $k$. Note that $\Sigma_{k}$ and $\Lambda_{k}$ are invariant under small deformations of $\eta$, as any deformation of a $d$ th power of a $(k / d)$-differential remains a $d$ th power.

The stratum $\Omega^{k} \mathcal{M}_{g}(\mu)$ is locally modeled on the periods of $E_{1}(\widehat{\Sigma} \backslash \widehat{P}, \widehat{Z})$ as discussed in Section 2. The support of the eigenspace $E_{i}(\widehat{\Lambda})$ for $i=0,1$ is on the preimage $\widehat{\Lambda}_{k}=\pi^{-1}\left(\Lambda_{k}\right)$, as the preimage of every simple closed curve in $\Lambda \backslash \Lambda_{k}$ has fewer than $k$ components. Since by Proposition 5.1, the $\mathrm{g}$ lobal residue condition only imposes conditions to nodes that correspond to $\widehat{\Lambda}_{k}$, we define spaces of residue assignments at such nodes

$$
R_{k}^{\text {grc }} \subseteq R_{k}^{\text {res }} \subseteq \mathbb{C}^{e^{0}\left(\widehat{\Lambda}_{k}\right)} \subseteq \mathbb{C}^{h^{0}\left(\widehat{\Lambda}_{k}\right)}
$$

The subspace $\mathbb{C}^{e^{0}\left(\widehat{\Lambda}_{k}\right)}$ consists of residue assignments that are equivariant under the deck transformation $\tau$. The subspace $R_{k}^{\text {res }}$ is further cut out by imposing the residue theorem to the lifts of the components in $\Sigma_{k}$ that are not local maxima of $\bar{\Gamma}$ and have no poles of order at least $k$ in the interior, whose number we denote by $c_{k, \mathrm{~L}}$. The subspace $R_{k}^{\text {grc }}$ moreover satisfies the global 


\section{BAINBRIDGE ET AL.}

residue condition (4-ab). As in (6.1), we obtain that

$$
e^{0}\left(\widehat{\Lambda}_{k}\right)-\operatorname{dim} R_{k}^{\mathrm{res}}=c_{k, \mathrm{~L}} .
$$

The space of (lifts to the normalized covers of) twisted $k$-differentials compatible with $\bar{\Gamma}$ except for the $\widehat{g}$ lobal residue condition is modeled on $E_{1}\left(\widehat{\Sigma} \backslash\left\{\widehat{P} \cup \widehat{\Lambda}^{\circ}\right\}, \widehat{\Lambda}^{+} \cup \widehat{Z}\right)$. Since the residue map from this space is dominant to $R_{k}^{\text {res }}$ and equidimensional over its image, it suffices to show that

$$
e_{1}\left(\widehat{\Sigma} \backslash\left\{\widehat{P} \cup \widehat{\Lambda}^{\circ}\right\}, \widehat{\Lambda}^{+} \cup Z\right)-e_{1}(\widehat{\Sigma} \backslash \widehat{P}, \widehat{Z})=\operatorname{dim} R_{k}^{\mathrm{res}}-\operatorname{dim} R_{k}^{\mathrm{grc}}
$$

as in (6.2).

Since by assumption, $\widehat{\Sigma}$ is connected, $h_{2}(\widehat{\Sigma} \backslash \widehat{P}, \widehat{Z})=h^{0}(\widehat{\Sigma} \backslash \widehat{Z}, \widehat{P})$ is zero or 1 . Note that if it is 1 , the non-trivial contribution comes from the class of $\widehat{\Sigma}$ itself, which is fixed under $\tau$. Hence, the $\zeta$-eigenspace of $H_{2}(\widehat{\Sigma} \backslash \widehat{P}, \widehat{Z})$ is trivial, which implies that $e_{2}(\widehat{\Sigma} \backslash \widehat{P}, \widehat{Z})=0$.

Now, the rest of the calculation is the same as in the abelian case, after decorating all symbols with hats and using respective eigenspaces. We thus conclude that

$$
e_{1}\left(\widehat{\Sigma} \backslash\left\{\widehat{P} \cup \widehat{\Lambda}^{\circ}\right\}, \widehat{\Lambda}^{+} \cup \widehat{\Lambda}^{-} \cup \widehat{Z}\right)-e_{1}(\widehat{\Sigma} \backslash \widehat{P}, \widehat{Z})=c_{k}
$$

as in (6.4), where $c_{k}:=e_{2}(\widehat{\Sigma} \backslash \widehat{P}, \widehat{\Lambda} \cup \widehat{Z})=e^{0}(\widehat{\Sigma} \backslash\{\widehat{\Lambda} \cup \widehat{Z}\}, \widehat{P})$ is the number of components in $\Sigma_{k}$ without poles of order at least $k$ in the interior, and the analogue of $\delta_{P}$ is $e_{2}(\widehat{\Sigma} \backslash \widehat{P}, \widehat{Z})=0$, as shown above. Then we have

$$
\operatorname{dim} R_{k}^{\mathrm{grc}}=e^{0}\left(\widehat{\Lambda}_{k}\right)-c_{k}
$$

as in (6.5), and moreover

$$
e_{1}\left(\widehat{\Sigma} \backslash\left\{\widehat{P} \cup \widehat{\Lambda}^{\circ}\right\}, \widehat{\Lambda}^{+} \cup \widehat{\Lambda}^{-} \cup \widehat{Z}\right)-e_{1}\left(\widehat{\Sigma} \backslash\left\{\widehat{P} \cup \widehat{\Lambda}^{\circ}\right\}, \widehat{\Lambda}^{+} \cup \widehat{Z}\right)=c_{k}-c_{k, \mathrm{M}}
$$

as in (6.6), where $c_{k, \mathrm{M}}:=e_{2}\left(\widehat{\Sigma} \backslash\left\{\widehat{P} \cup \widehat{\Lambda}^{\circ}\right\}, \widehat{\Lambda}^{+} \cup \widehat{Z}\right)=e^{0}\left(\widehat{\Sigma} \backslash\left\{\widehat{Z} \cup \widehat{\Lambda}^{\circ}\right\}, \widehat{\Lambda}^{-} \cup \widehat{P}\right)$ is the number of components in $\Sigma_{k}$ that are local maxima of $\bar{\Gamma}$ with no poles of order at least $k$, either in the interior or at the boundary. Since $c_{k}=c_{k, \mathrm{M}}+c_{k, \mathrm{~L}}$, we thus obtain the desired result as before.

As a consequence of the above calculation, we also obtain the following interpretation of the global residue condition, once we fix the set of components $\Sigma_{k}$ on which the $k$-differentials are $k$ th powers of abelian differentials:

$$
\operatorname{dim} R_{k}^{\mathrm{res}}-\operatorname{dim} R_{k}^{\mathrm{grc}}=c_{k, \mathrm{M}} .
$$

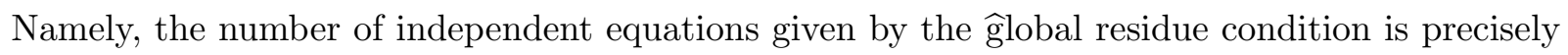
the number $c_{k, \mathrm{M}}$ of local maxima of $\bar{\Gamma}$ in $\Sigma_{k}$ with no poles of order at least $k$.

\section{Examples of flat geometric constructions with $k$-differentials}

In this section, we apply the incidence variety compactification to generalize certain flat geometric surgeries from abelian and quadratic differentials to $k$-differentials. Moreover, we sketch a flat geometric approach for proving Theorem 1.5.

EXAMPLE 7.1 (Breaking up a singularity). In this example, we use Theorem 1.5 to extend the notion of breaking up a zero of abelian and quadratic differentials (see [KZ03, Lan08]) to the case of $k$-differentials. Here, we describe the construction for singularities with $m>-k$. One can similarly extend this construction to the case of breaking up a pole of order at most $-r k$ into $r$ poles of order at most $-k$ or even more general settings. 


\section{StRATA OF $k$-DIFFERENTIALS}

Let $\left(X_{0}, \xi\right) \in \Omega^{k} \mathcal{M}_{g}\left(m, m_{1}, \ldots, m_{n}\right)$ be a $k$-differential with a singularity $z$ of order $m>-k$ that we want to break into $r$ singularities of orders $\widetilde{m}_{i}$, where $\sum_{i=1}^{r} \widetilde{m}_{i}=m$. Let $(X, \eta)$ be the twisted $k$-differential of type $\left(\widetilde{m}_{1}, \ldots, \widetilde{m}_{r}, m_{1}, \ldots, m_{n}\right)$, where $X$ is the union of $X_{0}$ with a $\mathbb{P}^{1}$ attached at $z$, the restriction $\left.\eta\right|_{X_{0}}$ equals $\xi$, and $\left.\eta\right|_{\mathbb{P}^{1}}$ is a $k$-differential with singularities of order $\widetilde{m}_{i}$ in the smooth locus of $\mathbb{P}^{1}$ and with a pole of order $m+2 k$ at the node $z$. If $(X, \eta)$ satisfies the global $k$-residue condition (4) in Definition 1.4, then the family of plumbed $k$-differentials in the proof of Theorem 1.5 breaks $z$ into $r$ singularities of orders $\widetilde{m}_{1}, \ldots, \widetilde{m}_{r}$ on smooth curves.

In this case, condition (4) does not hold if and only if $\xi$ is the $k$ th power of a holomorphic abelian differential and every differential in the stratum $\Omega^{k} \mathcal{M}_{0}\left(\widetilde{m}_{1}, \ldots, \widetilde{m}_{r},-2 k-m\right)$ has nonzero $k$-residue at the pole of order $2 k+m$. On the other hand, if there exists a differential in the stratum $\Omega^{k} \mathcal{M}_{0}\left(\widetilde{m}_{1}, \ldots, \widetilde{m}_{r},-2 k-m\right)$ with zero $k$-residue at the pole of order $2 k+m$, then the operation of breaking up such a singularity can be performed locally. Otherwise, we need to add a modification differential $\phi$ to $\xi$ as in the proof of Theorem 1.5, and hence the smoothing construction may fail to be local. For instance, this explains the failure of locally breaking up a zero of even degree into two zeros of odd degrees in the case of quadratic differentials, as remarked in [MZ08].

A consequence of the above discussion is that if $k \nmid m$, then one can always locally break up a singularity of order $m$ into $r$ singularities of orders that add up to $m$.

EXAMPLE 7.2 (Bubbling a handle). Another useful construction developed for abelian and quadratic differentials is bubbling a handle (see [KZ03, Lan08]). Given a quadratic differential $(X, q)$, this surgery increases the genus of $X$ by one and the order of a zero of $q$ by four while preserving the orders of the other singularities. Here, we generalize this operation to the case of $k$-differentials.

Let $\left(X_{0}, \xi\right) \in \Omega^{k} \mathcal{M}_{g}\left(m, m_{1}, \ldots, m_{n}\right)$ be a $k$-differential with a singularity $z$ of order $m>-k$ at which we want to bubble a handle. Let $(X, \eta)$ be the twisted $k$-differential of genus $g+1$ and of type $\left(m+2 k, m_{1}, \ldots, m_{n}\right)$, where $X$ is the union of $X_{0}$ with a torus $X_{1}$ attached at $z$, the restriction $\left.\eta\right|_{X_{0}}$ equals $\xi$, and $\left.\eta\right|_{X_{1}}$ is a $k$-differential that has a zero of order $m+2 k$ at some point of $X_{1}$ and a pole of order $-m-2 k$ at the node. If $(X, \eta)$ satisfies condition (4) in Definition 1.4, then the family of plumbed $k$-differentials obtained from $(X, \eta)$ gives the operation of bubbling a handle at $z$.

Note that there always exists a $k$-differential in the stratum $\Omega^{k} \mathcal{M}_{1}(m+2 k,-2 k-m)$ which has zero $k$-residue at the pole. If $k \nmid m$, the claim follows from definition. If $k \mid m$, we can simply take the $k$ th power of an abelian differential of type $(m / k+2,-m / k-2)$. Moreover, for every stratum different from $\Omega^{2} \mathcal{M}_{1}(4,-4)$, there exists a primitive differential in $\Omega^{k} \mathcal{M}_{1}(m+2 k,-2 k-m)$ with vanishing $k$-residue at the pole (see [GT17]). As a result, $(X, \eta)$ satisfies condition (4), and hence it is always possible to bubble a handle at such a singularity $z$ of a $k$-differential. This bubbling can furthermore be performed in such a way that the result is a primitive $k$-differential, even starting from the $k$ th power of an abelian differential - the only exception being trying to bubble a handle at a regular point of the square of an holomorphic differential.

EXAMPLE 7.3 (Flat geometric smoothing). As in the case of abelian differentials, the sufficiency of the conditions in Theorem 1.5 can also be explained by a flat geometric construction in the sense of a $(1 / k)$-translation structure, without passing to the canonical cover. We illustrate the idea via the following example. The reader may refer to [BCGGM18, Section 5] for more details on related objects that will be used below.

Let $(X, \eta)$ be a twisted $k$-differential compatible with a given level graph with two levels such 


\section{BAINBRIDGE ET AL.}

that the top level contains a single component $\left(X_{0}, \eta_{0}\right)$ and the lower level consists of several components $\left(X_{a}, \eta_{a}\right)$. Suppose that $\eta_{0}$ is the $(k / d)$ th power of a primitive $d$-differential. Denote the vertical nodes of $X$ by $q_{i}$, with $q_{i}^{-} \in X_{a(i)}$ in the lower level. On $X_{0}$, we want to insert a line segment representing $\operatorname{Res}_{q_{i}^{-}}^{k} \eta$ such that its middle point is at $q_{i}^{+}$. By this, we mean that the segment is one of the $k$ th roots of $\operatorname{Res}_{q_{i}^{-}}^{k} \eta$. If $d \neq k$, then we can choose any such roots. If $d=k$, we want to choose the roots such that the corresponding sum factor in equation (1.3) vanishes. Such a choice exists by the global $k$-residue condition. We divide each of these segments into $d$ segments of equal length $\tilde{r}_{i, j}$ and label the middle point of $\tilde{r}_{i, j}$ by $q_{i, j}$ for $1 \leqslant j \leqslant d$.

We next introduce the definition of a residue slit, as a collection of broken lines in the surface around which we will modify neighborhoods of size given by the $k$ th roots of the $k$-residues. Given the tuple $T=\left(\zeta^{j k / d} \tilde{r}_{i, j}\right)_{\{i, j\}}=\left(r_{1}, \ldots, r_{N}\right)$ with cardinality $N$, we fix a permutation $\pi \in S_{N}$ such that the slopes of $r_{\pi(1)}, \ldots, r_{\pi(N)}$ are monotone. Let $P=P(T, \pi)$ be the polygon whose edges are given by the vectors $r_{\pi(1)}, \ldots, r_{\pi(N)}$, consecutively. It follows that $P$ is convex. Let $B(P)$ be the barycenter of $P$. Let $p$ be a point in $X_{0}$, disjoint from the singularities of $\eta$. Shrinking $\eta_{i}$ if necessary, we place $P$ inside $X_{0}$ with $B(P)=p$. A $k$-residue slit for $(T, \pi)$ is then defined to be a collection of broken lines $\left\{b_{i, j}\right\}$ with the following properties:

- Each broken line $b_{i, j}$ starts from $q_{i, j}$ and connects to the middle point of the edge $\zeta^{j k / d} \tilde{r}_{i, j}$ of the polygon $P$. We denote by $b_{i, j}^{(\ell)}$ the $\ell$ th line segment of $b_{i, j}$ and by $\theta\left(b_{i, j}^{(\ell)}\right)$ the slope of $b_{i, j}^{(\ell)}$.

- The broken lines $b_{i, j}$ do not intersect each other, and they are disjoint from the singularities of $\eta$.

- The slopes $\theta\left(b_{i, j}^{(\ell)}\right)$ are different from those of $\pm \zeta^{j k / d} \tilde{r}_{i, j}$ for all $j \in\{0, \ldots, k / d-1\}$.

- The holonomy $h\left(b_{i, j}\right)$ associated with $b_{i, j}$ is $\zeta^{j k / d}$ with respect to the $(1 / k)$-translation structure of $X_{0}$.

For $t \in(0, \varepsilon)$ sufficiently small, we define the surface $X_{0, t}=X_{0, t}(T, \pi)$ obtained by modifying neighborhoods of size $t T$ around the residue slit as follows. Remove $P(T, \pi)$ from $X_{0}$. In the case that $\left\langle h\left(b_{i, j}^{(\ell)}\right)^{-1} \theta\left(b_{i, j}^{(\ell)}\right), \tilde{r}_{i, j}\right\rangle>0$, we remove neighborhood parallelograms swept out by planar segments with holonomy vector $t \cdot h\left(b_{i, j}^{(\ell)}\right) \tilde{r}_{i, j}$ centered along $b_{i, j}^{(\ell)}$ in general and glue the parallel sides of these removed parallelograms in pairs. On the other hand, for each of the segments where $\left\langle h\left(b_{i, j}^{(\ell)}\right)^{-1} \theta\left(b_{i, j}^{(\ell)}\right), \tilde{r}_{i, j}\right\rangle<0$, we add a parallelogram swept out by segments of holonomy $t \cdot h\left(b_{i, j}^{(\ell)}\right) \tilde{r}_{i, j}$ to the existing surface and glue the pieces as indicated in [BCGGM18, Figure 16].

Let us carry out the above construction in a simple but already interesting example. Consider the stratum $\Omega^{3} \mathcal{M}_{2}(3,3,1,-1)$, where the twisted cubic differential $(X, \eta)$ is given by $\left(X_{0}, \eta_{0}\right) \in$ $\Omega^{3} \mathcal{M}_{2}(3,3,1,0,-1)$ and $\left(X_{1}, \eta_{1}\right) \in \Omega^{3} \mathcal{M}_{0}(1,-1,-6)$ such that a marked ordinary point $q$ of $X_{0}$ is attached to the pole of order 6 of $X_{1}$. In particular, this example illustrates the operation of breaking up a regular point into a simple zero and a simple pole for cubic differentials. We present $\eta_{0}$ and $\eta_{1}$ by using (1/3)-translation structure along with the residue slit in Figure 4 .

In Figure 4 , the point $q$ coincide with $q_{0}$, and the segment representing the $k$-residue is the horizontal one. The polygon $P(T, \pi)$ is the equilateral triangle in dashed lines. The residue slit is pictured in dotted lines. The cubic differential obtained after gluing in the lower-level surface $t \eta_{1}$ at $q$ and opening up the residue slit is presented in Figure 5, where the unlabeled edge identifications are clear and the identifications denoted by 1 and 2 are given by composing translation with a rotation by $2 \pi / 3$. As $t \rightarrow 0$, that is, if we shrink the removed residue slit 
1
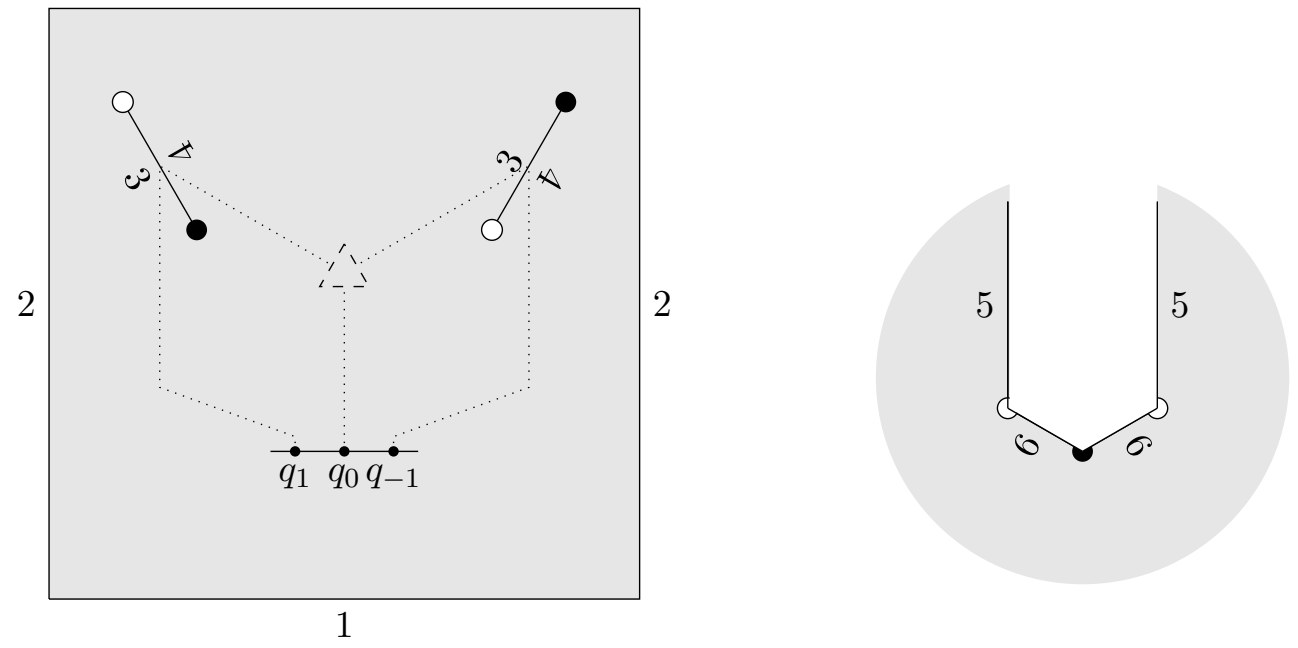

FiguRE 4. Flat geometric representation of a twisted cubic differential and the residue slit

neighborhood, the surface goes to $\left(X_{0}, \eta_{0}\right)$. Up to scaling, if alternatively we expand everything to arbitrarily large compared to the residue slit neighborhood, we then obtain $\left(X_{1}, \eta_{1}\right)$. A rigorous proof that justifies convergence in the sense of the incidence variety compactification can be found in [BCGGM18, Section 5.3].

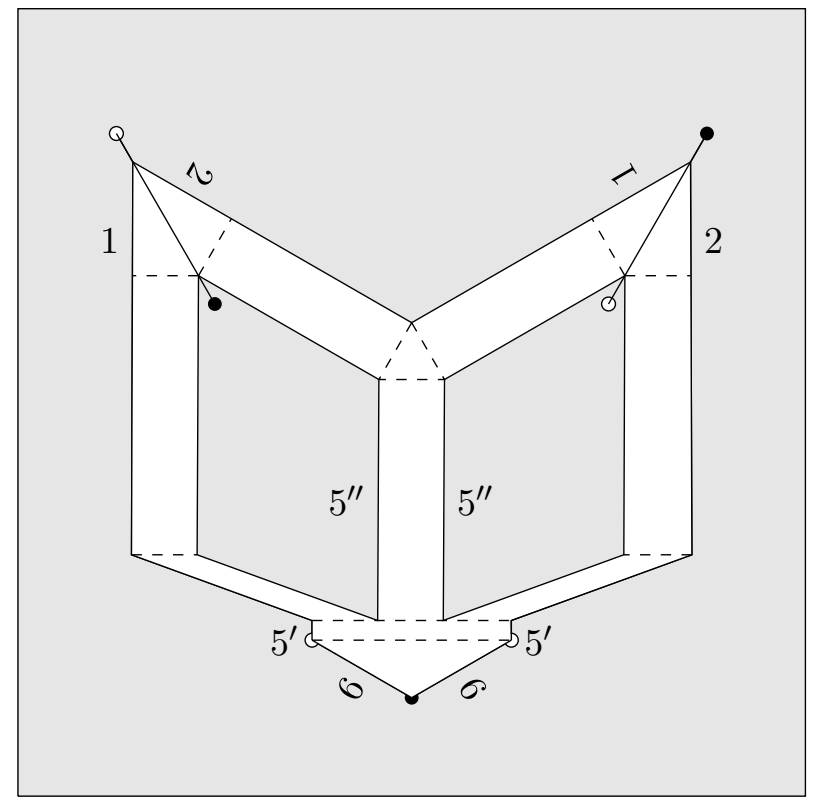

Figure 5. A plumbed surface in $\Omega^{3} \mathcal{M}_{2}(3,3,1,-1)$ 


\section{BAINBRIDGE ET AL.}

\section{ACKNOWLEDGEMENTS}

We thank the Casa Matemática Oaxaca (CMO), the Mathematisches Forschungsinstitut Oberwolfach (MFO), the Institute for Computational and Experimental Research in Mathematics (ICERM), and the Max Planck Institut für Mathematik, Bonn (MPIM), where various subsets of the authors met and substantial progress on this work was made. We are grateful to the organizers of the conferences for the invitations to participate. We also thank the anonymous referee for many valuable comments and suggestions, in particular leading to the previously missing point (iii) of Definition 1.4.

\section{REFERENCES}

BCGGM18 M. Bainbridge, D. Chen, Q. Gendron, S. Grushevsky, and M. Möller, Compactification of strata of abelian differentials, Duke Math. J. 167 (2018), no. 12, 2347-2416; doi:10.1215/00127094-2018-0012.

Boi15 C. Boissy, Connected components of the strata of the moduli space of meromorphic differentials, Comment. Math. Helv. 90 (2015), no. 2, 255-286; doi:10.4171/CMH/353.

Che17 D. Chen, Teichmüller dynamics in the eyes of an algebraic geometer, Surveys on Recent Developments in Algebraic Geometry (eds I. Coskun, T. de Fernex, and A. Gibney), Proc. Sympos. Pure Math., vol. 95 (Amer. Math. Soc., Providence, RI, 2017), 171-197.

EV92 H. Esnault and E. Viehweg, Lectures on vanishing theorems, DMV Seminar, vol. 20 (Birkhäuser Verlag, Basel, 1992); doi:10.1007/978-3-0348-8600-0.

GT17 Q. Gendron and G. Tahar, Différentielles à singularités prescrites, 2017, arXiv: 1705.03240.

HM79 J. Hubbard and H. Masur, Quadratic differentials and foliations, Acta Math. 142 (1979), no. 3-4, 221-274; doi:10.1007/BF02395062.

HM98 J. Harris and I. Morrison, Moduli of curves, Grad. Texts in Math., vol. 187 (Springer-Verlag, New York, 1998); http://dx.doi.org/10.1007/b98867.

HM82 J. Harris and D. Mumford, On the Kodaira dimension of the moduli space of curves (with an appendix by William Fulton), Invent. Math. 67 (1982), no. 1, 23-88; doi:10.1007/BF01393371.

Kod05 K. Kodaira, Complex manifolds and deformation of complex structures, Classics Math. (Springer-Verlag, Berlin, 2005); doi:10.1007/b138372.

KZ03 M. Kontsevich and A. Zorich, Connected components of the moduli spaces of Abelian differentials with prescribed singularities, Invent. Math. 153 (2003), no. 3, 631-678; doi:10.1007/s00222-003-0303-x.

Lan04 E. Lanneau, Hyperelliptic components of the moduli spaces of quadratic differentials with prescribed singularities, Comment. Math. Helv. 79 (2004), no. 3, 471-501; doi:10.1007/s00014-004-0806-0.

Lan08_ Connected components of the strata of the moduli spaces of quadratic differentials, Ann. Sci. École Norm. Supér. (4) 41 (2008), no. 1, 1-56; doi:10.24033/asens. 2062.

Möl08 M. Möller, Linear manifolds in the moduli space of one-forms, Duke Math. J. 144 (2008), no. 3, 447-487; doi:10.1215/00127094-2008-041.

Mon17 G. Mondello, On the cohomological dimension of the moduli space of Riemann surfaces, Duke Math. J. 166 (2017), no. 8, 1463-1515; doi:10.1215/00127094-0000004X.

MZ08 H. Masur and A. Zorich, Multiple saddle connections on flat surfaces and the principal boundary of the moduli spaces of quadratic differentials, Geom. Funct. Anal. 18 (2008), no. 3, 919-987; doi:10.1007/s00039-008-0678-3.

Sch18 J. Schmitt, Dimension theory of the moduli space of twisted k-differentials, Doc. Math. 23 (2018), 871-894; doi:10.25537/dm.2018v23.871-894. 
Spa66 E. H. Spanier, Algebraic topology (McGraw-Hill Book Co., New York - Toronto, Ont. - London, 1966).

Str84 K. Strebel, Quadratic differentials, Ergeb. Math. Grenzgeb. (3), vol. 5 (Springer-Verlag, Berlin, 1984); doi:10.1007/978-3-662-02414-0.

Vee86 W. A. Veech, The Teichmüller geodesic flow, Ann. of Math. 124 (1986), no. 3, 441-530; doi:10.2307/2007091.

Wri15 A. Wright, Translation surfaces and their orbit closures: an introduction for a broad audience, EMS Surv. Math. Sci. 2 (2015), no. 1, 63-108; doi:10.4171/EMSS/9.

Zor06 A. Zorich, Flat surfaces, Frontiers in Number Theory, Physics, and Geometry. I (Springer, Berlin, 2006), 437-583; doi:10.1007/978-3-540-31347-2_13.

Matt Bainbridge mabainbr@indiana.edu

Department of Mathematics, Indiana University, Bloomington, IN 47405, USA

Dawei Chen dawei.chen@bc.edu

Department of Mathematics, Boston College, Chestnut Hill, MA 02467, USA

School of Mathematics, Institute for Advanced Study, 1 Einstein Drive, Princeton, NJ 08540, USA

Quentin Gendron gendron@mpim-bonn.mpg.de

Institut für algebraische Geometrie, Leibniz Universität Hannover, Welfengarten 1, 30167 Hannover, Germany

Current address: Centro de Ciencias Matemáticas-UNAM, Antigua Car. a Pátzcuaro 8701, Col. Ex Hacienda San José de la Huerta, Morelia, Mich., México

Samuel Grushevsky sam@math.stonybrook.edu

Mathematics Department, Stony Brook University, Stony Brook, NY 11794-3651, USA

Martin Möller moeller@math.uni-frankfurt.de

Institut für Mathematik, Goethe-Universität Frankfurt, Robert-Mayer-Str. 6-8, 60325 Frankfurt am Main, Germany 